\title{
EFFECTIVE ESTIMATES ON INDEFINITE TERNARY FORMS
}

\author{
ELON LINDENSTRAUSS AND GREGORY MARGULIS \\ Dedicated to the memory of Joram Lindenstrauss
}

\begin{abstract}
We give an effective proof of a theorem of Dani and Margulis regarding values of indefinite ternary quadratic forms at primitive integer vectors. The proof uses an effective density-type result for orbits of the groups $\mathrm{SO}(2,1)$ on $\mathrm{SL}(3, \mathbb{R}) / \mathrm{SL}(3, \mathbb{Z})$.
\end{abstract}

\section{INTRODUCTION}

1.1. In 1929 A. Oppenheim conjectured that if $Q$ is an indefinite quadratic form in $d \geq 5$ variables then

$$
\inf \left\{|Q(v)|: v \in \mathbb{Z}^{d} \text { primitive }\right\}=0 .
$$

For rational indefinite forms $(1.1 \mathrm{a})$ is equivalent to the classical Meyer theorem that a rational indefinite quadratic form in $d \geq 5$ variables represents 0 over $\mathbb{Z}$ nontrivially. Let us remember that for $d=3,4$ there are examples of rational indefinite quadratic forms in $d$ variables which do not represent 0 over $\mathbb{Z}$ nontrivially.

Later it was realized that if $Q$ is an irrational form (i.e., is not proportional to a quadratic form with rational coefficients) then (1.1a) should remain true even if $d=3,4$. On the other hand, it is well-known that 1.1a is false for many indefinite irrational binary quadratic forms. Note that the conjecture becomes easier as $d$ gets larger: by restricting a quadratic form in $d$ variables to an appropriate $d^{\prime}<d$ dimensional rational subspace it is easy to deduce the conjecture for $d$ variables from the case of $d^{\prime}$ variable.

1.2. Partial results for this conjecture were proved using analytical methods, notably the Hardy-Littlewood Circle Method and its variants; in particular Davenport and Heilbronn $[\mathrm{DH}$ established the conjecture for indefinite diagonal forms $Q\left(x_{1}, \ldots, x_{5}\right)=\sum_{i} \lambda_{i} x_{i}^{2}$, where $\lambda_{1}, \ldots, \lambda_{5}$ are nonzero real numbers not all of the same sign. For the more difficult case of the general forms progress was slower; combining results by Birch, Davenport and Ridout the Oppenheim Conjecture was established for forms in $d \geq 21$ variables by the late 1950's, and

Date: August 9, 2013.

E. L. was supported in part by the ERC (AdG Grant 267259) and the NSF (DMS-0800345). G. M. was supported in part by the NSF (DMS-0801195). 
despite some improvement this was still the state-of-the-art in the mid 1980's.

1.3. In the mid-seventies, M.S.Raghunathan made the insightful observation that the Oppenheim Conjecture would follow from a conjecture about closures of orbits of unipotent subgroups. The Raghunathan conjecture states that if $G$ is a connected Lie group, $\Gamma$ a lattice in $G$ (that is, $\Gamma$ is a discrete subgroup such that $G / \Gamma$ carries a $G$-invariant probability measure), and $U$ a connected Ad-unipotent subgroup of $G$ (that is, $\operatorname{Ad} u$ is a unipotent linear transformation for any $u \in U$ ), then for any $x \in G / \Gamma$ there exists a closed connected subgroup $L=L(x)$ such that the closure of the orbit $U . x$ coincides with L.x (unipotent orbit rigidity). In a more general form of Raghunathans conjecture, the connected subgroup $U$ is not necessarily unipotent but generated by unipotent elements.

1.4. Inspired by the above-mentioned observation of M.S. Raghunathan, the second named author proved the Oppenheim Conjecture in full generality (i.e. for $d \geq 3$ ) in the mid-eighties $[\mathrm{M} 3, \mathrm{M} 2]$. The corresponding dynamical statements, which is equivalent to the Oppenheim Conjecture and proved in [M3, M2], says that any bounded orbit of $H=\mathrm{SO}(2,1)$ in $\mathrm{SL}(3, \mathbb{R}) / \overline{\mathrm{SL}}(3, \mathbb{Z})$ is closed; it is interesting to note that in implicit form this equivalence already appeared in a paper by Cassels and Swinnerton-Dyer CSD . This dynamical statement can be considered as a special case of Raghunathan's conjecture in its more general form given above.

In [DM1] Dani and Margulis proved that the orbits of $H$ in $G / \Gamma$ for $G=\mathrm{SL}(3, \mathbb{R})$ and $\Gamma=\mathrm{SL}(3, \mathbb{Z})$ are either closed or dense. In [DM2] the same authors proved the Raghunathan conjecture also in the more involved case where $U$ is a one-parameter unipotent subgroup of $H$ acting on $G / \Gamma$.

1.5. In full generality, the Raghunathan conjecture was proved in 1990 by Ratner [R5] using a different approach. The proof in [R5] is based on an equidistribution theorem for unipotent flows, also proved in R5] and uses the countability of certain set of subgroups of $G$ depending on $\Gamma$. The equidistribution theorem can be considered as the quantitative strengthening of the unipotent orbit rigidity for one-parameter unipotent subgroup, and it says that if $\left\{u_{t}: t \in \mathbb{R}\right\}$ is a one-parameter Ad-unipotent subgroup of $G$ and $x \in G / \Gamma$ then there exists a homogeneous probability measure $\mu_{x}$ on $G / \Gamma$ with $x$ in its support such that

$$
\frac{1}{T} \int_{0}^{T} f\left(u_{t} \cdot x\right) d t \rightarrow \int f(y) d \mu_{x}(y) \quad \text { as } T \rightarrow \infty
$$

for every bounded continuous function $f$ on $G / \Gamma$ (a measure $\mu$ on $G / \Gamma$ is called homogeneous if there exists a closed subgroup $F$ of $G$ such 
that $\mu$ is F-invariant and $\operatorname{supp} \mu=F$.y for some $y \in G / \Gamma)$. This equidistribution theorem was conjectured by Dani in [D2] for the case $G / \Gamma=\operatorname{SL}(n, \mathbb{R}) / \operatorname{SL}(n, \mathbb{Z})$ and for the general case in $\mid \mathrm{M} 4$.

The hardest part of the proof of the equidistribution theorem is the proof of unipotent measure rigidity which Ratner proved in a series of three papers $[\mathrm{R} 3, \mathrm{R} 2, \mathrm{R} 4]$. The unipotent measure rigidity was conjectured by Dani in $[\mathrm{D} 1]$, and it says that any finite $U$-ergodic $U$-invariant measure $\mu$ on $G / \Gamma$ is homogeneous where $U$ is a one-parameter Adunipotent subgroup of $U$. Ratner's early works on horocycle flow, particularly her classification of joinings in [R1], can be viewed in this context as special cases of this much more general measure classification.

A shorter and arguably more conceptual proof of unipotent measure rigidity was given by Margulis and Tomanov (see [MT1 for the case where $G$ is algebraic and [MT2] for a simple reduction to that case).

1.6. A refined version of the equidistribution theorem was proved in [DM4]. As in [R5], the proof in [DM4] also relies on the classification of invariant measures, i.e. unipotent measure rigidity. Other crucial ingredients in the proof of the equidistribution theorem, both in R5] and [DM4] are nondivergence of orbits of unipotent flows regarding the amount of time and orbits can spend outside of large compact subsets in $G / \Gamma$ (see $\$ 4$ for details) and an "avoidability" argument explaining why an orbit cannot spend too much time near certain proper subvarieties of $G / \Gamma$. The avoidability argument are proved differently in [R5] and [DM4]; the method of [DM4 based on the use of finite dimensional representations of $G$ is known as the linearization technique.

These equidistribution results, which rely on the classification of invariant measures, are of great intrinsic interest. However, if one is only interested in Raghunathan's conjecture it seems feasible to give such a proof which is much closer in spirit to the original approach of [M3, M2, DM1, DM2]. In particular, for $G / \Gamma$ a product of $\operatorname{SL}\left(2, K_{i}\right) / \Gamma_{i}$ a direct proof of orbits rigidity along these lines was given by N. Shah in $[\mathrm{S}]$.

1.7. A weaker version of the main number theoretic result in DM1 is the following:

1.8. Theorem (Dani-Margulis). Let $Q$ be an indefinite, irrational, ternary quadratic form. Then the set

$$
\left\{Q(v): v \in \mathbb{Z}^{3} \text { primitive }\right\}
$$

is dense in the real line.

1.9. The main result of this paper is the following quantification of Theorem 1.8. We implicitly assume all integral quadratic forms we consider are primitive in the sense that they are not a nontrivial integer multiple of another integral quadratic form. 
1.10. Theorem. Let $Q_{1}$ be an indefinite, ternary quadratic form with $\operatorname{det} Q_{1}=1$ and $\epsilon>0$. Then for any $T \geq T_{0}(\epsilon)\left\|Q_{1}\right\|^{K_{1}}$ at least one of the following holds:

(i) There is an integral quadratic form $Q_{2}$ with $\left|\operatorname{det}\left(Q_{2}\right)\right|<T^{\epsilon}$ and

$$
\left\|Q_{1}-\lambda Q_{2}\right\| \ll\left\|Q_{1}\right\| T^{-1} \quad \text { where } \lambda=\left|\operatorname{det}\left(Q_{2}\right)\right|^{-1 / 3} \text {. }
$$

(ii) For any $\xi \in\left[-(\log T)^{\kappa_{2}},(\log T)^{\kappa_{2}}\right]$ there is a primitive integer vector $v \in \mathbb{Z}^{3}$ with $0<\|v\|<T^{K_{3}}$ satisfying

$$
\left|Q_{1}(v)-\xi\right| \ll(\log T)^{-\kappa_{2}}
$$

(with $K_{1}, \kappa_{2}, K_{3}$, and the implicit constants absolute).

1.11. We note that proving the inhomogeneous approximation above, i.e. that in a ball of radius $T^{K_{3}}$ one can find primitive integral vectors $v$ for which $\left|Q_{1}(v)-\xi\right|$ is small for any $\xi$ in an interval, entails a substantial complication in comparison to the corresponding homogeneous question of showing that there is such a vector $v$ with $\left|Q_{1}(v)\right|$ small.

Another feature worth noting is the quality of the approximation: if one is content with an estimate of the form $\left\|Q_{1}(v)-\xi\right\| \ll(\log \log T)^{-1}$ the combinatorial apparatus of $\$ 9$, perhaps the most technical section in this paper, is not needed.

Since by Liouville's Theorem algebraic numbers cannot be too well approximated by rationals, we can conclude the following from Theorem 1.10 .

1.12. Corollary. Let $Q_{1}$ be a reduced, indefinite, ternary quadratic form which is not proportional to an integral form but has algebraic coefficients. Then for any $T \geq T_{0}\left(Q_{1}\right)$ (with $T_{0}$ depending effectively on the degrees and heights of the coefficients of $\left.Q_{1}\right)$, for any

$$
\xi \in\left[-(\log T)^{\kappa_{2}},(\log T)^{\kappa_{2}}\right]
$$

there is a primitive integer vector $v \in \mathbb{Z}^{3}$ with $0<\|v\|<T^{K_{3}}$ satisfying

$$
\left|Q_{1}(v)-\xi\right| \ll(\log T)^{-\kappa_{2}}
$$

with $\kappa_{2}, K_{3}$ as in Theorem 1.10 .

1.13. Theorem 1.8 is only part of what is proved in [DM1]. While it is possible to give an effective and quantitative version of the full force of the main result of [DM1] with our methods, the resulting bounds would be substantially worse than what we give above.

1.14. An important difference between the analytic methods used to tackle the Oppenheim Conjecture and the dynamical proof of e.g. $\mathrm{M} 2$, M3. DM1 is that the analytic proofs are effective in the sense that they provide an upper bound on the size of the shortest integer vector $v$ for which $|Q(v)|<\epsilon$, while at least on the face of it dynamical proofs provided no such bound. For instance, while this is not explicated in their paper, the proof of Davenport and Heilbronn for the Oppenheim 
Conjecture for irrational indefinite forms of the type $Q(q)=\lambda_{1} x_{1}^{2}+$ $\cdots+\lambda_{5} x_{5}^{2}$ gives an upper bound on the size of such an individual integer vector $v$ in terms of Diophantine properties of the coefficients $\lambda_{i}$; assuming e.g. $\lambda_{1} / \lambda_{2}$ is Diophantine generic (i.e. there are $c, C$ so that $\left|\lambda_{1} / \lambda_{2}-p / q\right| \geq c q^{-C}$ for all rational numbers $\left.p / q\right)$ this upper bound is polynomial in $\epsilon^{-1}$.

Bentkus and Götze gave in [BG1] an analytic proof, with effective estimates, of the Oppenheim conjecture for general indefinite quadratic forms in $d \geq 9$ variables, and more recently, Götze and Margulis [GM] have been able to give an analytic proof, with effective error estimates, of the Oppenheim Conjecture for general indefinite quadratic forms in $d \geq 5$ variable; we note that 5 seems to be a natural barrier to the applicability of such techniques.

1.15. The proof of the Oppenheim conjecture in [M3, M2] and a simplified proof in DM3 uses the existence of minimal invariant sets for actions of groups on compact spaces; formally these proofs depend on the axiom of choice. Dani in [D4] gave a proof of the Oppenheim conjecture based on the existence of a recurrent point as a substitute to working with minimal sets; in particular, his proof is independent of the axiom of choice.

The proof of the Raghunathan conjecture in [R5] uses the unipotent measure rigidity (see above) and the proof of the unipotent measure rigidity uses the ergodic decomposition and the pointwise ergodic theorem for essentially arbitrary invariant measures, which seems to us to be harder to effectivize than the existence of minimal sets.

1.16. Though there are significant differences, the strategy which we use in our paper has many similarities with the strategy which was used by Margulis in [M3, M2 and subsequent papers by Dani and Margulis [DM1, DM2, DM3]. The main ingredient in these strategies is to prove that an orbit closure contains orbits of additional subgroups. In the papers quoted, this is achieved using minimal sets for appropriately chosen subactions, while in our paper the beginning point of the orbit of the new subgroup is moving. To make this approach work, we need to control how this base point changes so it remains sufficiently generic in an appropriate quantitative sense.

Acknowledgements. This work began with discussions of the authors with A. Venkatesh at the IAS in Princeton in 2005-2006. We thank Venkatesh for sharing with us his insights, and the IAS for its hospitality. During the long time it has taken us to write these results we have started to collaborate with Amir Mohammadi and Nimish Shah on giving an effective proof to the general orbit closure classification theorem. We thank Amir Mohammadi and Nimish Shah for 
many helpful discussions. We would also like to thank the university of Beilefeld for its hospitality during July 2010.

This paper is dedicate to the memory of Joram Lindenstrauss, E.L.'s father, who passed away on April 29, 2012.

\section{Statement of DyNAMical Results}

2.1. Let $G=\operatorname{SL}(3, \mathbb{R}), \Gamma=\operatorname{SL}(3, \mathbb{Z})$, and e the identity element of $G$. If $L<G$ we shall denote by $[g]_{L}$ the image of $g \in G$ under the natural map $G \rightarrow G / L$. We identify the space $X=G / \Gamma$ with the space of unit volume lattices in $\mathbb{R}^{3}$ by identifying $[g]_{\Gamma} \in X$ with the lattice $g \mathbb{Z}^{3}$ in $\mathbb{R}^{3}$.

2.2. Let $H=\mathrm{SO}(2,1)<G$, which we view as the subgroup of $G$ preserving the quadratic form $Q_{0}(x, y, z)=y^{2}-2 x z$. The following subgroups of $G$ will play a special role in the proof:

$$
\begin{array}{rlrl}
D=\{a(t): t \in \mathbb{R}\}<H & a(t) & =\left(\begin{array}{ccc}
e^{t} & & \\
& 1 & \\
& & e^{-t}
\end{array}\right) \\
U=\{u(s): s \in \mathbb{R}\}<H & u(s)=\left(\begin{array}{ccc}
1 & s & \frac{s^{2}}{2} \\
& 1 & s \\
& & 1
\end{array}\right) \\
U^{-}=\left\{u^{-}(s): s \in \mathbb{R}\right\}<H & u^{-}(s)=\left(\begin{array}{ccc}
1 & & \\
s & 1 & \\
\frac{s^{2}}{2} & s & 1
\end{array}\right) \\
V=\{v(s): s \in \mathbb{R}\} \nless H & v(s)=\left(\begin{array}{ccc}
1 & & s \\
& 1 & \\
& & 1
\end{array}\right) .
\end{array}
$$

We shall use the notations $u_{t}$ and $u(t)$ etc. interchangeably. Note that $D, U, U^{-}<H$ while $V \cap H=\{\mathbf{e}\}$.

2.3. Theorem 1.8 as well as the other number theoretic results of [DM1] were obtained from the following theorem regarding the action of $H$ on $X$ :

2.4. Theorem (Dani and Margulis). For any $x \in X$, the orbit H.x is either periodic or dense.

Note that by H.x periodic we mean that it is closed and supports a finite $H$-invariant measure. As mentioned above, Theorem 2.4 is a special case of a general conjecture of Raghunathan that was later proved by Ratner in [R5]. In [DM3] a substantially more elementary proof of Theorem 1.8 (and hence the Oppenheim Conjecture) was given based in particular on the observation that the following weaker result suffices for proving Theorem 1.8 . 
2.5. Theorem (Dani and Margulis). For any $x \in X$, if H.x is not periodic then there is a point $y \in \overline{H . x}$ so that V.y $\subset \overline{H . x}$.

2.6. In order to state an effective version of Theorem 2.5, we need to be able to measure the complexity of the periodic orbits $H . x$, to play a similar role in the statement of an effective version of this theorem to that played by the determinant of an integral quadratic form in Theorem 1.10.

There are several reasonable choices how to measure the complexity of the periodic $H$-orbit $H . x$ : for instance, one can consider the volume of $H . x$ or equivalently the covolume of the stabilizer of $x$ in $H$.

Another logical choice is measuring the arithmetic complexity of $H . x$ using the discriminant as defined in [ELMV] and [EMV, §17.3]). As shown in [EMV, Prop. 17.4], one can bound both from above and below each of these invariants of periodic $H$-orbits by the other.

Yet a third way of measuring the complexity of the periodic $H$-orbits is through the connection between such orbits and integral quadratic forms. Indeed, if $H \cdot[g]_{\Gamma}$ is periodic, then $g^{-1} H g \cap \Gamma$ is a lattice in $g^{-1} \mathrm{Hg}$, and hence in particular is Zariski dense there. Therefore, up to multiplicative scalar, there is a unique quadratic form invariant under $g^{-1} \mathrm{Hg} \cap \Gamma$, namely $Q_{0} \circ g$. Since the elements of $g^{-1} \mathrm{Hg} \cap \Gamma$ are integral there is a $s_{0} \in \mathbb{R}$ so that $Q_{2}=s_{0} Q_{0} \circ g$ is integral and primitive. We set the quadratic discriminant of a periodic orbit $H .[g]_{\Gamma}$, denoted by $\operatorname{disc}_{Q}\left(H \cdot[g]_{\Gamma}\right)$, to be $\left|\operatorname{det} Q_{2}\right|=\left|s_{0}\right|^{3}$.

Since in this paper our main motivation is the study of quadratic forms it seems to reasonable to use this height as a measure of complexity. In any case, it is quite easy to bound the discriminant of a periodic orbit H.x as defined in [ELMV, EMV] polynomially above and below by $\operatorname{disc}_{Q}(H . x)$, so for the purposes of this paper the discriminant and the quadratic discriminant are essentially the same.

2.7. In addition to having a way of measuring the complexity of periodic $H$-orbits, we need to choose how to measure the size of an element $h \in H$ (or $\in G)$. We shall use both the Euclidean norm $\|\cdot\|$ and the $\ell^{\infty}$-norm $\|\cdot\|_{\infty}$ on $\mathbb{R}^{3}$, and use the (Euclidean) operator norm on $M_{3}(\mathbb{R})$ (in particular on the Lie algebra $\mathfrak{g}$ of $G$ ). Let

$$
B_{T}^{H}=\{h \in H:\|h-\mathbf{e}\|<T\} .
$$

Note the use of the operator norm, and not a Riemannian metric, in the definition of these sets; we will also use at times a right invariant Riemannian metric $d_{G}$ on $G$, and the corresponding metric (simply denoted by $d$ ) on $G / \Gamma$.

2.8. Theorem. Let $\epsilon, \eta \in(0,1)$ and $x_{1} \in X_{\eta}$. Then for any $T>$ $T_{0}(\epsilon) \eta^{-K_{4}}$ (with $K_{4}$ an absolute constant), at least one of the following holds: 
(i) there is a point $x_{2} \in G / \Gamma$ with $H . x_{2}$ periodic and with

$$
\operatorname{disc}_{Q}\left(H . x_{2}\right)<T^{3 \epsilon}
$$

so that $d\left(x_{1}, x_{2}\right) \leq T^{-1}$

(ii) there is a $h \in B_{T^{K_{5}}}^{H_{5}}$ so that $h . x_{1} \in X_{\kappa_{6}}$ and so that for every $s \in\left[-(\log T)^{\kappa_{7}},(\log T)^{\kappa_{7}}\right]$ the point $v(s) h . x_{1}$ is within $(\log T)^{-\kappa_{7}}$ of a point in the set $B_{T^{K_{5}}}^{H} . x_{1}$ with $K_{5}, \kappa_{6}, \kappa_{7}$ absolute constants.

2.9. The two cases given by this theorem are not mutually exclusive. Indeed, Einsiedler, Margulis and Venkatesh [EMV] have proved a general quantitative equidistribution result (with polynomial rates) for periodic orbits of semisimple groups. An explicit version for orbits of semisimple subgroups $H$ which are maximal in $G$, which is the case we are interested in, was given by Mohammadi [M5]. The main results [EMV, Thm. 1.3] or [M5, Thm. 1.1] of these papers show that the periodic $H$-orbits occurring in (i) satisfy (ii) as long as $\operatorname{disc}_{Q}\left(H . x_{2}\right) \gg$ $(\log T)^{c}$, and hence by employing these results Theorem 2.8 can be somewhat strengthened. The proof of these theorems from [EMV, M5] is much less elementary than the techniques of this paper, relying in particular on uniform spectral gap estimates for congruence subgroups which can be attained by combining Selberg's estimates on the Fourier coefficients of modular forms and the Jacquet-Langlands correspondence. We prefer to state the theorem as above in order to keep this paper self-contained and elementary.

A remark about notations. we use $c_{i}, C_{i}, \kappa_{i}, K_{i}$ to denote most of the constants appearing in this paper. $c_{i}$ and $\kappa_{i}$ will denote small constants, i.e. constants that need to be taken to be smaller than something depending on all previously chosen constants, and $C_{i}$ and $K_{i}$ large constants in the corresponding sense. The paragraphs are numbered, and the constants $c_{i}, C_{i}$ are numbered per paragraph. The constants $\kappa_{i}, K_{i}$ on the other hand, are global, and retain their meaning throughout the paper. While we have not evaluated the various constants involved our argument is quite explicit (indeed, we have made an effort to keep it so!) and in principle the reader should have no difficulty evaluated them if she or he would so desire.

\section{Overview of the Proof of Theorem 2.8}

3.1. Our proof of Theorem 2.8 gives a new proof of Theorem 2.5. We begin by presenting the steps in this new proof, then explain how the statements need to be modified for a quantitative proof.

We split the proof of Theorem 2.5 into two parts, first finding two points in an orbit closure of a nonperiodic $H$-orbit that differ by an element of $V$, and then using the dynamics along $U$ to get additional points on a $V$ orbit. Both of these ingredients appear in the original proof (though in the context of studying minimal orbit closures), but by 
switching the order we can avoid some of the more intricate arguments in e.g. DM3 needed to control the relative position of two points on which we apply the $U$-action.

3.2. Proposition. Let $x_{1} \in X$ be such that H. $x_{1}$ is not periodic. Then for any $t>0$ there is a

$$
x_{2} \in v(t) \overline{H \cdot x_{1}} \cap v(-t) . \overline{H \cdot x_{1}}
$$

with $x_{2} \notin\left[P_{i}\right]_{\Gamma}$ for $P_{i}$ one of the two parabolic groups

$$
P_{1}=\left(\begin{array}{lll}
* & * & * \\
0 & * & * \\
0 & * & *
\end{array}\right) \quad \text { or } \quad P_{2}=\left(\begin{array}{ccc}
* & * & * \\
* & * & * \\
0 & 0 & *
\end{array}\right) \text {. }
$$

Note that the conditions that $x_{2} \notin\left[P_{1}\right]_{\Gamma}$ is equivalent to requiring that the lattice in $\mathbb{R}^{3}$ corresponding to $x_{2}$ does not contain a $U$-fixed vector, and $x_{2} \notin\left[P_{1}\right]_{\Gamma}$ is equivalent to the lattice in $\mathbb{R}^{3}$ corresponding to $x_{2}$ not having a 2-dimensional rational subspace fixed by $U$. Theorem 2.5 now follows from the following:

3.3. Proposition. Let $t>0$ and $x_{2} \in X \backslash\left(\left[P_{1}\right]_{\Gamma} \cup\left[P_{2}\right]_{\Gamma}\right)$. Then there is a $x_{3} \in \overline{U . x_{2}} \cap Y$ so that

$$
v([-t, t]) \cdot x_{3} \subset \overline{D\{v(-t), v(t)\} U \cdot x_{2}}
$$

with $Y \subset X$ a fixed compact set.

3.4. Given these two propositions, it is easy to conclude the proof of Theorem 2.5. Indeed, choose $t_{i} \rightarrow \infty$, and for each $i$ find using Proposition 3.2 a point

$$
x_{2}^{(i)} \in v\left(t_{i}\right) \overline{H \cdot x_{1}} \cap v\left(-t_{i}\right) \overline{H \cdot x_{1}}
$$

$\underline{\text { with }} x_{2}^{(i)} \notin\left[P_{1}\right]_{\Gamma} \cup\left[P_{2}\right]_{\Gamma}$. Now apply Proposition 3.3 to find $x_{3}^{(i)} \in$ $\overline{U . x_{2}^{(i)}} \cap Y$ so that

$$
v\left(\left[-t_{i}, t_{i}\right]\right) \cdot x_{3}^{(i)} \subset \overline{D\left\{v\left(-t_{i}\right), v\left(t_{i}\right)\right\} U \cdot x_{2}^{(i)}} \subset \overline{H \cdot x_{1}},
$$

with the second inclusion a consequence of $3.4 \mathrm{a}$; indeed, since $U$ and $V$ commute

$$
\overline{D v\left(t_{i}\right) U \cdot x_{2}^{(i)}}=\overline{D U \cdot\left(v\left(t_{i}\right) \cdot x_{2}^{(i)}\right)} \subset \overline{D U H \cdot x_{1}}=\overline{H \cdot x_{1}}
$$

and similarly with $-t_{i}$ replacing $t_{i}$.

Since all $x_{3}^{(i)}$ lie in the same compact set $Y$, there is a convergent subsequence, and it is clear that if $x_{3}^{(\infty)}$ is a limit point of the $x_{3}^{(i)}$ then

$$
V x_{3}^{(\infty)} \subset \overline{H \cdot x_{1}} .
$$


3.5. In order to give a quantitative and effective proof, one needs a quantitative substitute to the qualitative condition $x \notin\left[P_{1}\right]_{\Gamma} \cup\left[P_{2}\right]_{\Gamma}$.

For $x=[g]_{\Gamma} \in X$, let

$$
\begin{aligned}
& \alpha_{1}(x)^{-1}=\min \left\{\|v\|_{\infty}: v \in g \mathbb{Z}^{3} \text { nonzero }\right\} \\
& \alpha_{2}(x)^{-1}=\min \left\{\|v \wedge w\|_{\infty}: v, w \in g \mathbb{Z}^{3} \text { linearly independent }\right\}
\end{aligned}
$$

where we define the $\ell^{\infty}$-norm on $\mathbb{R}^{3} \wedge \mathbb{R}^{3}$ in terms of the basis $e_{i} \wedge e_{j}$ of $\mathbb{R}^{3} \wedge \mathbb{R}^{3}$ with $e_{i}$ denoting the standard basis of $\mathbb{R}^{3}$. Let $\alpha(x)=$ $\max \left(\alpha_{1}(x), \alpha_{2}(x)\right)$, and for any $\kappa>0$ let

$$
X_{\kappa}=\left\{x \in X: \alpha(x) \leq \kappa^{-1}\right\} .
$$

These form an increasing sequence of compact sets whose union is $X$.

Note that $x \in\left[P_{1}\right]_{\Gamma}$ if and only if the corresponding lattice contains a vector of the form $(c, 0,0)^{\top}$; such a vector is contracted exponentially by $a(-t)$ - indeed,

$$
a(-t)\left(\begin{array}{l}
c \\
0 \\
0
\end{array}\right)=\left(\begin{array}{c}
c e^{-t} \\
0 \\
0
\end{array}\right)
$$

and hence if $x \in\left[P_{1}\right]_{\Gamma}$ one has that $\alpha_{1}(a(-t) x) \gg e^{t}$; it is not hard to see that the converse also holds. Similarly, $x \in\left[P_{2}\right]_{\Gamma}$ iff $\alpha_{2}(a(-t) x) \gg$ $e^{t}$ as $t \rightarrow \infty$. As a substitute to $X \backslash\left(\left[P_{1}\right]_{\Gamma} \cup\left[P_{2}\right]_{\Gamma}\right)$ we shall make use of the following:

3.6. Definition. For any $\delta, \kappa \in(0,1)$ and $k \in \mathbb{R}_{\geq 0} \cup\{\infty\}$, set

$$
\Xi(k, \kappa, \delta)=\left\{x \in X: \forall j \in \mathbb{Z} \cap[0, k], a(-j) . x \in X_{\kappa e^{-\delta j}}\right\} .
$$

We extend this definition to all $t \in \mathbb{R}$ by defining $\Xi(k, \kappa, \delta)=X$ for $k<0$.

We can now state the two results used to prove Theorem 2.8,

3.7. Proposition. Let $\delta, \eta, \epsilon \in(0,1)$ and $x_{1} \in X_{\eta}$. Then for any

$$
T>T_{0}(\delta, \epsilon) \eta^{-K_{8}}
$$

(with $K_{8}$ absolut $\oint^{(1)}$ and $T_{0}$ effectively computable in terms of these parameters), at least one of the following holds:

(i) There is a point $x_{2} \in G / \Gamma$ so that $H . x_{2}$ is periodic and

$$
\begin{aligned}
& \operatorname{disc}_{Q}\left(H . x_{2}\right)<T^{3 \epsilon} \\
& d_{G / \Gamma}\left(x_{1}, x_{2}\right)<T^{-1} .
\end{aligned}
$$

(ii) For any $\tau \in\left[1, T^{\kappa_{9} \epsilon}\right]$ there are $h, h^{\prime} \in B_{T^{K_{10}}}^{H}, x^{\prime} \in \Xi\left(\kappa_{11} \epsilon \log T, \kappa_{12}, \delta\right)$ and $\tau^{\prime} \in[\tau / 2, \tau]$ so that

$$
\begin{array}{r}
d_{G / \Gamma}\left(v(-\tau) \cdot x^{\prime}, h \cdot x_{1}\right)<c_{1} T^{-\kappa_{13} \epsilon} \\
d_{G / \Gamma}\left(v(\tau) \cdot x^{\prime}, h^{\prime} \cdot x_{1}\right)<c_{1} T^{-\kappa_{13} \epsilon}
\end{array}
$$

\footnotetext{
${ }^{(1)}$ Explicitly, $K_{8}$ can be taken to be 11 .
} 
with $K_{10}$ an absolute constant and $c_{1}, \kappa_{9}, \kappa_{11}, \kappa_{12}, \kappa_{13}$ depending only on $\delta$.

Note that the constant $K_{4}$ of Theorem 2.8 can be taken to be equal to $K_{8}$ above.

3.8. Theorem. Fix $\delta, \kappa \in(0,1)$. Then for any $T>T_{0}(\delta)$, if

$$
x_{1} \in \Xi(\log (T / \kappa), \kappa, \delta) \quad t \in\left(0,(\log T)^{\kappa_{14}}\right),
$$

there is a $s \in[-T, T]$ so that $x_{3}=u(s) . x_{2} \in \Xi\left(\frac{1}{2} \log T, \tilde{\kappa}, \delta\right)$ and so that for every $\xi \in v([-t, t]) . x_{3}$ there is a

$$
\xi^{\prime} \in B_{(\log T)^{K_{15}}}^{D}\{v(-t), v(t)\} u([-T, T]) \cdot x_{3}
$$

with $d\left(\xi, \xi^{\prime}\right)<(\log T)^{\kappa_{14}}$; here $\tilde{\kappa}, \kappa_{14}, K_{15}$ depend only on $\delta$.

Proposition 3.7 and Theorem 3.8 for a single value of $\delta \in(0,1)$ clearly imply Theorem 2.8 . We state (and prove) them for all $\delta \in(0,1)$ since this gives a more pleasing effective analogue of Proposition 3.2 and Proposition 3.3 respectively.

3.9. In [R2], as a step in the proof of measure rigidity for unipotent flows, Ratner proves in particular that in the context we consider here if $\mu$ is a $U$-invariant and ergodic probability measure on $G / \Gamma$ then either the measures $a_{-t} \mu$ escape to the cusp as $t \rightarrow \infty$, or $\mu$ is invariant under a conjugate of $H$ by $V$. While the context of her result is different, it has a somewhat similar flavour to our use of $\Xi(k, \kappa, \delta)$.

\section{INHERITABLE BOUNDEDNESS CONDITIONS}

4.1. An important ingredient of the proof is the use of an appropriate Diophantine condition that can be efficiently used in the main inductive lemma. Recall that for any $\delta, \kappa \in(0,1)$ we let

$$
\Xi(k, \kappa, \delta)=\left\{x \in X: \forall j \in \mathbb{Z} \cap[0, k], a(-j) . x \in X_{\kappa e^{-\delta j}}\right\} .
$$

4.2. Using the results on quantitative nondivergence, we show in this section that the boundedness condition above is inherited for most points on any $u_{t}$-orbit.

We shall use a quantitative nondivergence estimate from $[\overline{\mathrm{KM}}]$; this estimate due to Kleinbock and Margulis is based on [M1] and its modification by Dani in [D3. The following follows directly from KM, Theorem 5.2] for the special case of $u_{t}$-orbits (and $u_{t}^{-}$-orbits) on $X$; as in $\overline{\mathrm{KM}}$ it will be convenient for us to work with $\ell^{\infty}$-norm on $\mathbb{R}^{3}$ as well as on $\mathbb{R}^{3} \wedge \mathbb{R}^{3}$ (with respect to the basis $e_{i} \wedge e_{j}$ with $e_{i}$ the standard basis of $\mathbb{R}^{3}$ ).

4.3. Proposition. Let $x \in X, T>0$ be such that

(1) for any $v \neq 0$ in the lattice corresponding to $x$ in $\mathbb{R}^{3}$,

$$
\max _{t \in[0, T]}\left\|u_{t} v\right\|_{\infty} \geq 1
$$


(2) for any linearly independent vectors $v, w$ in the lattice corresponding to $x$,

$$
\max _{t \in[0, T]}\left\|u_{t}(v \wedge w)\right\|_{\infty} \geq 1
$$

Then for any $\epsilon \in(0,1)$

$$
m\left(\left\{t \in[0, T]: u_{t} . x \notin X_{\epsilon}\right\}\right) \leq K_{16} \epsilon^{1 / 2} T,
$$

where $m$ denotes Lebesgue measure and $K_{16}$ an absolute constant. Exactly the same statement also holds for $u_{t}^{-}$.

The exponent here is $\frac{1}{2}$ since for any vectors $v, w \in \mathbb{R}^{3}$ the functions $\left\|u_{t} v\right\|_{\infty},\left\|u_{t}(v \wedge w)\right\|_{\infty}$ are the maximum of polynomials of degree $\leq 2$ and hence in the notations of $[\overline{\mathrm{KM}}]$ are $\left(C, \frac{1}{2}\right)$-good for some $C$ (cf. [KM, Lemm. 3.1 \& Prop. 3.2]).

4.4. Proposition. Let $\delta, \kappa, \tilde{\kappa} \in(0,1), k \geq 0$, and $T \geq e^{k}$. Then for any point $x \in \Xi(k, \kappa, \delta)$

$$
m\left(\left\{t \in[0, T]: u_{t} \cdot x \notin \Xi((1-\delta) k+\log (\kappa)-10, \tilde{\kappa}, \delta)\right\}\right) \leq \frac{K_{16} T \tilde{\kappa}^{1 / 2}}{1-e^{-\delta / 2}}
$$

with $K_{16}$ an absolute constant.

Proof. Set $\epsilon_{j}=\kappa e^{-\delta j}, \tilde{\epsilon}_{j}=\tilde{\kappa} e^{-\delta j}$. By Proposition 4.3, for all $j \in$ $\{0, \ldots,\lfloor(1-\delta) k+\log (\kappa)\rfloor-10\}$

$$
m\left(\left\{t \in\left[0, e^{-j} T\right]: u_{t} a_{-j} . x \notin X_{\tilde{\epsilon}_{j}}\right\}\right) \leq K_{16} \tilde{\epsilon}_{j}^{1 / 2} e^{-j} T
$$

unless there is some $j$ in this range and a one or two dimensional sub-lattice of $a_{-j} . x$ corresponding to a vector $p \in \mathbb{R}^{3}$ or $\wedge^{2} \mathbb{R}^{3}$ with

$$
\left\|u_{t} p\right\|_{\infty}<1
$$

throughout the interval $t \in\left[0, e^{-j} T\right]$. It follows that unless there are such $j, p$, equation 4.4a holds.

In order to complete the proof of the proposition, it suffice to show that the existence of such $j, p$ is in contradiction to $x \in \Xi(k, \kappa, \delta)$. Suppose first $p=\sum_{i=1}^{3} p_{i} e_{i} \in \mathbb{R}^{3}$. Then $p$ satisfies $\left\|u_{t} p\right\|_{\infty}<1$ throughout the interval $t \in\left[0, T e^{-j}\right]$, hence $\left|p_{3}\right|<1$ and for all $t$ in this range

$$
\begin{aligned}
\left|p_{1}+t p_{2}+t^{2} p_{3} / 2\right| & <1 \\
\left|p_{2}+t p_{3}\right| & <1 .
\end{aligned}
$$

It follows that $\left|p_{1}\right|<1,\left|p_{2}\right|<C_{1} e^{j} T^{-1}$ and $\left|p_{3}\right|<C_{1}^{2} e^{2 j} T^{-2}$, with e.g. $C_{1}=e^{5}$. Then since $T \geq e^{k}$ and $j \leq k-\delta k+\log \kappa-10$ we have $T \geq C_{1} \epsilon_{k}^{-1} e^{j+1}$ and hence

It follows that

$$
\left\|a_{-\lceil\delta k-\log \kappa\rceil} p\right\|_{\infty}<\kappa e^{-\delta k}=\epsilon_{k} .
$$

$$
a_{-j-\lceil\delta k-\log \kappa\rceil} . x \notin X_{\epsilon_{k}}
$$

and as $j+\lceil\delta k-\log \kappa\rceil<k$ this is in contradiction to $x \in \Xi(k, \kappa, \delta)$. 
A similar argument holds if $p \in \mathbb{R}^{3} \wedge \mathbb{R}^{3}$. Write $p=p_{12} e_{1} \wedge e_{2}+$ $p_{13} e_{1} \wedge e_{3}+p_{23} e_{2} \wedge e_{3}$. Then $e_{1} \wedge e_{2}$ spans the $u_{t}$-invariant subspace of $\mathbb{R}^{3} \wedge \mathbb{R}^{3}$, and

$$
u_{t} p=\left(p_{12}+t p_{13}+\frac{t^{2}}{2} p_{23}\right) e_{1} \wedge e_{2}+\left(p_{13}+t p_{23}\right) e_{2} \wedge e_{3}+p_{23} e_{2} \wedge e_{3}
$$

and the situation is entirely analogous to the previous case .

In order to produce points in $\Xi(k, \kappa, \delta)$ starting from an arbitrary initial point $x$ we need to use the flow in the $U^{-}$direction:

4.5. Proposition. Let $x \in X_{\eta}$ for $0<\eta<1$, and $T \geq K_{17}$. Then

$$
m\left(\left\{t \in[0, T]: a(\log \eta) u_{t}^{-} \cdot x \notin \Xi(\infty, \kappa, \delta)\right\}\right) \leq \frac{K_{16} \kappa^{1 / 2} T}{1-e^{-\delta / 2}} .
$$

Proof. The proof is very similar to that of Proposition 4.4. Set $\epsilon_{j}=$ $\kappa e^{-\delta j}$. Applying again Proposition 4.3, we see that for all $j \geq 0$

$$
m\left(\left\{t \in\left[0, e^{j} \eta^{-1} T\right]: u_{t}^{-} a(-j+\log \eta) . x \notin X_{\epsilon_{j}}\right\}\right) \leq K_{16} \epsilon_{j}^{1 / 2}
$$

unless there is some $j \geq 0$ and a one or two dimensional sub-lattice of $a_{-j+\log \eta} . x$ corresponding to a vector $p \in \mathbb{R}^{3}$ or $\wedge^{2} \mathbb{R}^{3}$ with

$$
\left\|u_{t}^{-} p\right\|_{\infty}<1
$$

throughout the interval $t \in\left[0, e^{j} \eta^{-1} T\right]$. It follows that unless there are such $j, p$, equation (4.5a) holds.

In order to complete the proof of the proposition, it suffice to show that the existence of such $j, p$ is in contradiction to $x \in X_{\eta}$. Suppose first $p=\sum_{i=1}^{3} p_{i} e_{i} \in \mathbb{R}^{3}$. Then $p$ satisfies $\left\|u_{t}^{-} p\right\|_{\infty}<1$ throughout the interval $t \in\left[0, e^{j} T\right]$. A similar calculation to that given in the proof of Proposition 4.4 shows that $\left|p_{1}\right|<C_{1}^{2} T^{-2} e^{-2 j} \eta^{2},\left|p_{2}\right|<C_{1} T^{-1} e^{-j} \eta$ and $\left|p_{3}\right|<1$ for $C_{1}=e^{5}$, hence if $T>e^{10} \eta^{-1}$

$$
\begin{aligned}
\alpha_{1}(x)^{-1} \leq\|a(j-\log \eta) p\|_{\infty} & =\max \left(\eta^{-1} e^{j}\left|p_{1}\right|,\left|p_{2}\right|, \eta e^{-j}\left|p_{3}\right|\right) \\
& <e^{-j} \eta
\end{aligned}
$$

in contradiction to $x \in X_{\eta}$.

The case of $p \in \wedge^{2} \mathbb{R}^{3}$ is similar. 
To state the following lemmas, we need to name a few more subgroups of $G$ :

$$
\begin{aligned}
P & =\left\{\left(\begin{array}{lll}
* & * & * \\
& * & * \\
& & *
\end{array}\right)\right\} \quad \mathcal{U}=[P, P] \\
P^{-} & =\left\{\left(\begin{array}{lll}
* & \\
* & * & \\
* & * & *
\end{array}\right)\right\} \quad \mathcal{U}^{-}=\left[P^{-}, P^{-}\right] \\
A & =\left\{\left(\begin{array}{lll}
t & & \\
& s & \\
& & (s t)^{-1}
\end{array}\right): s, t \in \mathbb{R}^{*}\right\}=P \cap P^{-} .
\end{aligned}
$$

4.6. Lemma. Let $g \in G$ satisfy that $h_{0} a g=g \gamma_{0}$ for $h_{0} \in B_{\kappa_{18}}^{G}$, a $\in$ $B_{\kappa_{18}}^{A}$ and $\gamma_{0} \in \Gamma \backslash\{\mathbf{e}\}$ for sufficiently small absolute constant $\kappa_{18}<1$ (cf. (2.7a) for the definition of $B_{\kappa}^{A}$ ). Then $\gamma_{0}$ is unipotent and moreover at least one of the following two possibilities hold:

(1) $\gamma_{0}$ is not a generic unipotent element (i.e. $\left.\left(\gamma_{0}-\mathbf{e}\right)^{2}=0\right)$

(2) the lattice $[g]_{\Gamma}$ contains a nontrivial vector $v$ with

$$
\|v\| \leq K_{19}\left\|h_{0}-\mathbf{e}\right\| \text {. }
$$

Proof. We begin by choosing $\kappa_{18}$ so that if $\left\|g_{i}-\mathbf{e}\right\|<\kappa_{18}$ for $i=1,2$ then

$$
\left|\operatorname{tr}\left(g_{1} g_{2}\right)-3\right|,\left|\operatorname{tr}\left(\left(g_{1} g_{2}\right)^{2}\right)-3\right|<\frac{1}{2} .
$$

Since for every $\gamma \in \Gamma$ it holds that $\operatorname{tr}(\gamma) \in \mathbb{Z}$, it follows that if $\|a-\mathbf{e}\|,\left\|h_{0}-\mathbf{e}\right\|<\kappa_{18}$ then $\gamma_{0}$, hence $h_{0} a$ which is conjugate to $\gamma_{0}$, is unipotent. It follows that

$$
\left(h_{0} a-\mathbf{e}\right)^{3}=0 .
$$

Write $h_{0}=u_{+} a_{0} u_{-}$with $a_{0} \in A$ and $u_{ \pm} \in \mathcal{U}^{ \pm}$; since multiplication gives a local diffeomorphism $\mathcal{U}^{+} \times A \times \mathcal{U}^{-} \rightarrow G$ near e

$$
\left\|h_{0}-\mathbf{e}\right\| \asymp \max \left(\left\|u_{+}-\mathbf{e}\right\|,\left\|a_{0}-\mathbf{e}\right\|,\left\|u_{-}-\mathbf{e}\right\|\right) .
$$

By equation 4.6a) (e.g. by applying $\left(h_{0} a-\mathbf{e}\right)^{3}$ to the standard basis of $\mathbb{R}^{3}$ ) it follows that

$$
\left\|a_{0} a-\mathbf{e}\right\| \leq C_{1} \max \left(\left\|u_{+}-\mathbf{e}\right\|,\left\|u_{-}-\mathbf{e}\right\|\right)
$$

from which we conclude that

$$
\left\|h_{0} a-\mathbf{e}\right\| \leq C_{2}\left\|h_{0}-\mathbf{e}\right\| .
$$

Suppose now that $\left(\gamma_{0}-\mathbf{e}\right)^{2} \neq 0$ (or equivalently, $\left(h_{0} a-\mathbf{e}\right)^{2} \neq 0$ ). Then conjugating $\gamma_{0}$ by an element of $\Gamma$ if necessary, we can assume that $\gamma_{0}$ has the form

$$
\gamma_{0}=\left(\begin{array}{ccc}
1 & n_{1} & n_{2} \\
& 1 & n_{3} \\
& & 1
\end{array}\right) \quad \text { with } n_{1}, n_{3} \neq 0
$$


Let $e_{1}, e_{2}, e_{3}$ be the standard basis to $\mathbb{R}^{3}$. Then as $h_{0} a=g \gamma_{0} g^{-1}$, equation $4.6 \mathrm{~b}$ implies that

$$
\begin{aligned}
\left\|g e_{1}\right\| & =\left|n_{1}\right|^{-1}\left\|g \gamma_{0} e_{2}-g e_{2}\right\| \\
& \leq\left\|g \gamma_{0} g^{-1}-\mathbf{e}\right\|\left\|g e_{2}\right\| \leq C_{2}\left\|h_{0}-\mathbf{e}\right\|\left\|g e_{2}\right\| .
\end{aligned}
$$

As $\left\|h_{0}-\mathbf{e}\right\| \leq \kappa_{18}$, if $\kappa_{18}$ is sufficiently small, by replacing $\gamma_{0}$ by $p^{-1} \gamma_{0} p$ and $g$ by $g p$ for suitable $p \in \Gamma \cap P$, we may assume that the inner product $\left(g e_{1}, g e_{2}\right)$ satisfies

$$
\left(g e_{1}, g e_{2}\right)<\left\|g e_{1}\right\|\left\|g e_{2}\right\| / 100 .
$$

A similar argument shows that

$$
\left\|g e_{2}\right\| \leq C_{3}\left\|h_{0}-\mathbf{e}\right\|\left\|g e_{3}\right\|
$$

and that without loss of generality $\left(g e_{i}, g e_{3}\right)<\left\|g e_{i}\right\|\left\|g e_{3}\right\| / 100$ for $i=1,2$.

Since $\operatorname{det} g=1$, the above bounds on $\left(g e_{i}, g e_{j}\right)$ imply

$$
\left\|g e_{1}\right\|\left\|g e_{2}\right\|\left\|g e_{3}\right\|<2 .
$$

Using the estimates $4.6 \mathrm{c}$ and $4.6 \mathrm{~d}$ we conclude that

$$
\left\|g e_{1}\right\|^{3}<C_{4}\left\|h_{0}-\mathbf{e}\right\|^{3},
$$

and hence $v=g e_{1}$ is a nontrivial vector in $[g]_{\Gamma}$ with $\|v\| \ll\left\|h_{0}-\mathbf{e}\right\|$.

4.7. Lemma. Let $x \in \Xi(k, \kappa, \delta)$, with $\delta<1$ and $\kappa>0$. Then for any $s$ with $K_{20}<|s|<\kappa_{21} e^{(1-\delta) k}$, if $h_{0}=u_{+} a_{0} u_{-} \in B_{\kappa_{18}}^{G}$ satisfies $u(s) . x=h_{0} \cdot x$, with $u_{+} \in \mathcal{U}^{+}, u_{-} \in \mathcal{U}^{-}, a_{0} \in A$, then

$$
\left\|u_{-}-\mathbf{e}\right\| \geq \kappa_{22}|s|^{-\frac{2+\delta}{1-\delta}} .
$$

with $\kappa_{18}$ an absolute constant, and $K_{20}, \kappa_{21}, \kappa_{22}$ depending only on $\kappa$.

Proof. Write $x=[g]_{\Gamma}$ with $g \in G$; Then by definition

$$
u(s) g=h_{0} g \gamma
$$

and clearly if $s$ is large, the matrix $\gamma \neq \mathbf{e}$. In fact, we can say a bit more: if $s$ is large (say $s>K_{20}$ ), the matrix $\gamma$ will not satisfy $(\gamma-\mathbf{e})^{2}=0$, since it is conjugate to the matrice $h_{0}^{-1} u(s)$, and $h_{0}^{-1} u(s)$ cannot have two orthogonal fixed vectors $\left(h_{0}\right.$ is in some fixed neighborhood of $\mathbf{e}$, and for any given $C>0$, if $s$ is large enough, the collection of vectors $v$ for which $\|u(s) v\|<C\|v\|$ lie in a narrow cone in $\mathbb{R}^{3}$ ).

Let $0 \leq t \leq k$ be an integer to be determined later, and write $g_{1}=a(-t) g$. Then

$$
u\left(e^{-t} s\right) g_{1}=a(-t) u(s) g=a(-t) h_{0} a(t) g_{1} \gamma .
$$

It follows that $h_{1} a_{0} g_{1}=g_{1} \gamma^{-1}$ for

$$
h_{1}=\left(a(-t) u(s) u_{+} a(t)\right) \cdot\left(a_{0} a(-t) u_{-} a(t) a_{0}^{-1}\right) ;
$$


note that

$$
\begin{aligned}
\left\|h_{1}-\mathbf{e}\right\| & \leq C_{1}\left(e^{-t}|s|+e^{-t}\left\|u_{+}-\mathbf{e}\right\|+e^{2 t}\left\|u_{-}-\mathbf{e}\right\|\right) \\
& \leq C_{2}\left(e^{-t}|s|+e^{2 t}\left\|u_{-}-\mathbf{e}\right\|\right)
\end{aligned}
$$

Since $[g]_{\Gamma} \in \Xi(k, \kappa, \delta)$, we know that $\left[g_{1}\right]_{\Gamma} \in X_{\kappa e^{-\delta t}}$, and so any nontrivial vector in the lattice corresponding to $\left[g_{1}\right]_{\Gamma}$ has norm $\geq \kappa e^{-\delta t}$. Using Lemma 4.6 we conclude that

$$
\left\|h_{1}-\mathbf{e}\right\|>K_{19}^{-1} \kappa e^{-\delta t} .
$$

Combining (4.7a) and (4.7b) we obtain that

$$
\left(e^{-t}|s|+e^{2 t}\left\|u_{-}-\mathbf{e}\right\|\right)>c_{3} \kappa e^{-\delta t} .
$$

We now choose $t$ so that

$$
\frac{c_{3}}{6} \kappa e^{(1-\delta) t}<|s|<\frac{c_{3}}{2} \kappa e^{(1-\delta) t}
$$

as long as $K_{20}$ was chosen to be sufficiently big (depending only on $\kappa$ ) the condition on $|s|$ in the statement of lemma implies that $0 \leq t \leq k$. Then

$$
\left\|u_{-}-\mathbf{e}\right\|>\frac{c_{3}}{2} \kappa e^{-(2+\delta) t}
$$

and hence by $4.7 \mathrm{c}$ it follows that $\left\|u_{-}-\mathbf{e}\right\| \gg|s|^{-\frac{2+\delta}{1-\delta}}$.

\section{A Closing Lemma}

5.1. We first introduce a few more notations. Since $H$ is a simple Lie group, $\mathfrak{g}=$ Lie $G$ splits into a direct sum of Ad $H$-invariant summands, in this case given by $\mathfrak{g}=\mathfrak{h} \oplus \mathfrak{m}$ with $\mathfrak{h}$ the Lie algebra of $H$ and $\mathfrak{m}$ an irreducible 5-dimensional representation of $H$. We can further split $\mathfrak{m}$ into eigenspaces for Ad $D$, namely $\mathfrak{m}=\bigoplus_{i=-2}^{2} \mathfrak{m}_{i}$ with

$$
\operatorname{Ad} a(t)[\underline{v}]=e^{j t} \underline{v} \quad \text { for } \underline{v} \in \mathfrak{m}_{j} ;
$$

note that $\mathfrak{m}_{2}$ coincides with the Lie algebra $\mathfrak{v}$ of $V$.

5.2. Lemma (Closing Lemma). Let $M>0$ be arbitrary, $\delta, \kappa \in(0,1)$, $T \geq T_{0}(M, \delta, \kappa)$ and $x \in \Xi(\log T, \kappa, \delta)$. Then there are constants $\kappa_{23}$, $\kappa_{24}$ depending on $\delta$, and a constant $K_{25}$ depending on $M$ (explicitly, $\left.K_{25}=5 M+22\right)$ so that at least one of the following two possibilities holds:

(A) There is a periodic H-trajectory H. $x_{1}$ with $\operatorname{disc}_{Q}\left(H . x_{1}\right) \leq T^{60}$ and $d\left(x, x_{1}\right) \leq T^{-M}$.

(B) There exist $s, s^{\prime} \in[0, T]$ so that

$$
u(s) . x, u\left(s^{\prime}\right) \cdot x \in \Xi\left((1-\delta) \log T-\log (1 / \kappa)-10, \kappa_{23}, \delta\right)
$$

and in addition the point $u(s) \cdot x$ can be expressed as $u(s) \cdot x=$ $h \exp (\underline{m}) u\left(s^{\prime}\right) . x$ with $h \in H, \underline{m} \in \mathfrak{m}$ satisfying

$$
\|h-e\|<T^{-\kappa_{24}}, \quad T^{-K_{25}}<\|\underline{m}\|<T^{-\kappa_{24}}
$$


The following elementary facts will be useful in proving the Closing Lemma:

5.3. Lemma. Let $h_{1}, h_{2}$ be two non-commuting elements of $H$ of infinite order. Then up to scalar, $Q_{0}(x, y, z)=y^{2}-2 x z$ is the unique $\left\langle h_{1}, h_{2}\right\rangle$-invariant quadratic form.

The easy proof of Lemma 5.3 is left to the reader.

5.4. Lemma. Let $A$ be $a n \times m$-integer matrix and $B$ a $n \times m$ real matrix. Assume

$$
\|A-B\|\|A\|^{m-1}<\kappa_{26} .
$$

Suppose $v \in \mathbb{R}^{m}$ is a nonzero vector with $B v=0$. Then there exists a nonzero integer vector $v^{\prime} \in \mathbb{Z}^{m}$ with

(1) $A v^{\prime}=0$

(2) $\left\|v^{\prime} \wedge v\right\| \leq K_{27}\|A-B\|^{\frac{1}{m-1}}\|v\|$.

If in addition

$$
\operatorname{dim}\{w: A w=0\} \leq 1
$$

then

(3) $\left\|v^{\prime}\right\|<K_{28}\|A\|^{m-1}$.

Here $\kappa_{26}, K_{27}, K_{28}$ are constants depending only on $m$.

Proof. Let $\epsilon=\|A-B\|, \pi_{v}$ denote the orthogonal projection from $\mathbb{R}^{m}$ to the subspace $\mathbb{R} v$, and $\pi_{v}^{\prime}(w)=w-\pi_{v}(w)$ the complementary orthogonal projection. Consider all integral vectors in the ball $B_{T}(0)$ of radius $T=(3 \epsilon)^{-1}$ in $\mathbb{R}^{m}$. There are $\geq \beta(m) T^{m}-C_{1} T^{m-1}$ such vectors (with $\beta(m)$ the volume of the $m$-dimensional ball), and for any such vector $w, \pi_{v}^{\prime}(w)$ lies in a $m-1$-dimensional ball of radius $T$.

It follows that there will be distinct $w, w^{\prime} \in \mathbb{Z}^{m}$ with

$$
\left\|\pi_{v}^{\prime}\left(w-w^{\prime}\right)\right\|<2 r \quad r=2(\beta(m) T)^{-1 /(m-1)}
$$

since otherwise we would have $\geq \beta(m) T^{m}-C_{1} T^{m-1}$ disjoint balls of radius $r$ inside a ball of radius $T+r$ in $m-1$-dimensional space; the total volume of these balls would be at least

$$
\left(\beta(m) T^{m}-C_{1} T^{m-1}\right) \times \beta(m-1) r^{m-1}>\beta(m-1)(2 T)^{m-1}-C_{2} T^{m-2},
$$

which is greater than the total volume $\beta(m-1)(T+r)^{m-1}$ of the ball of radius $T+r$ if $\epsilon$ is small enough - in contradiction.

Let $v^{\prime}$ be a primitive integral vector with $w-w^{\prime} \in \mathbb{Z} v^{\prime}$, with $w, w^{\prime} \in$ $\mathbb{Z}^{m}$ as in the preceding paragraph. Then

$$
\left\|v^{\prime}\right\|<\frac{2}{3 \epsilon} \quad\left\|\pi_{v}^{\prime}\left(v^{\prime}\right)\right\|<C_{3} \epsilon^{1 /(m-1)}
$$


with $C_{3}=4(3 \beta(m))^{m-1}$. Since $B v=0$, it follows that

$$
\begin{aligned}
\left\|A v^{\prime}\right\| & \leq\|A\|\left\|\pi_{v}^{\prime}\left(v^{\prime}\right)\right\|+\|A-B\|\left\|\pi_{v}\left(v^{\prime}\right)\right\| \\
& \leq C_{3}\|A\| \epsilon^{1 /(m-1)}+\epsilon\left\|v^{\prime}\right\| \\
& \leq \frac{2}{3}+C_{3}\|A\| \epsilon^{1 /(m-1)} .
\end{aligned}
$$

If we now impose the condition that $\epsilon<\left(\frac{C_{3}}{3}\|A\|\right)^{-m+1}$ the integrality of $A$ and $v^{\prime}$ imposes that $A v^{\prime}=0$.

The last statement (3) of the lemma follows from Siegel's Lemma (or the pigeonhole principle). Explicitly, there are $\geq \beta(m)(T-1)^{m}$ integral vectors $w$ of size $\leq T$; if the image of $A$ is $m-1$-dimensional, then there are $\leq \beta(m-1)\|A\|^{m-1}(T+1)^{m-1}$ possibilities for $A w$; hence if $\beta(m)(T-1)^{m}>\beta(m-1)\|A\|^{m-1}(T+1)^{m-1}$, i.e. $T \geq C_{4}\|A\|^{m-1}$, there would be a nontrivial integer solution to $A v^{\prime \prime}=0$ with $\left\|v^{\prime \prime}\right\|<2 T$; by our assumption that the kernel of $A$ is one-dimensional and $v^{\prime}$ is primitive it follows that $v^{\prime \prime}$ is a multiple of $v^{\prime}$, hence $\left\|v^{\prime}\right\|<2 C_{5}\|A\|^{m-1}$.

5.5. We now proceed to prove Lemma 5.2. Unless otherwise specified the constants $c_{i}, C_{i}$ are allowed to depend on $\delta$ (and only on $\delta$ ). Assume that (B) does not hold (for $\kappa_{24}, K_{25}$ to be chosen later, but a good choice for $K_{25}$ would be $\left.5 M+22\right)$; we will show that this implies that (A) must hold. By Proposition 4.4, taking

$$
\kappa_{23}=\left(\frac{1-e^{-\delta / 2}}{2 K_{16}}\right)^{2}
$$

we have that the set $F \subset[0, T]$ defined by

$$
F=\left\{s \in[0, T]: u(s) . x \in \Xi\left((1-\delta) \log T-\log (1 / \kappa)-10, \kappa_{23}, \delta\right)\right\}
$$

satisfies that $m(F) \geq T / 2$. It follows that there is a $K$-separated subset $S \subset F$, i.e. a subset such that $\left|s-s^{\prime}\right| \geq K$ for every distinct $s, s^{\prime} \in S$, with $|S| \geq T / 2 K$, for $K \geq 1$ to be determined later in a way that depends on $\delta$ only. Since $\Xi\left(k-\log (1 / \kappa)-10, \kappa_{23}, \delta\right) \subset X_{\kappa_{23}}$, and the latter is a compact set (depending only on $\delta$ ) in a nice 8-dimensional space, it follows that there is a $T^{-\eta}$-neighborhood $O_{1}$ in $X$ containing at least $c_{1} T^{1-8 \eta}$ points from the set $\{u(s) . x: s \in S\}$. Here $\eta$ (which can be essentially identified with the constant $\kappa_{24}$ in the statement of the lemma) is a constant to be determined later according to $\delta$. Let

$$
S_{1}=\left\{s \in S: u(s) . x \in O_{1}\right\},
$$

and enumerate the points of $S_{1}$ in increasing order $s_{1}<\cdots<s_{N}$; in particular $N \geq c_{1} T^{1-8 \eta}$. Let $1 \leq j<N$ be such that $s_{j+1}-s_{j}$ is minimal; clearly

$$
s_{j+1}-s_{j} \leq \frac{1}{N}\left(s_{N}-s_{1}\right) \leq \frac{T^{8 \eta}}{c_{1}} .
$$


Fix $g \in G$ in some fixed compact lift of $X_{\kappa_{23}}$ satisfying

$$
[g]_{\Gamma}=u\left(s_{j}\right) \cdot x \text {. }
$$

For each $1 \leq i<N$ let $\gamma_{i} \in \Gamma$ be chosen so that $g \gamma_{i}$ is the closest point in $g \Gamma$ to $u\left(s_{i}-s_{j}\right) g$; write

$$
u\left(s_{i}-s_{j}\right) g=w_{i} g \gamma_{i} \quad \text { with }\left\|w_{i}-\mathbf{e}\right\|<T^{-\eta} .
$$

Note that since all $u\left(s_{i}\right) \cdot x$ are in the small neighborhood $O_{1}$ and $S$ is at least 1 -seperated, $\gamma_{i} \neq \mathbf{e}$ for all $i \neq j$ and also $\gamma_{i} \neq \gamma_{i^{\prime}}$ for $i \neq i^{\prime}$. Finally, note that by $(5.5 \mathrm{~b})$

$$
\left\|\gamma_{i}\right\|=\left\|g^{-1} w_{i}^{-1} u\left(s_{i}-s_{j}\right) g\right\|<C_{2}\left|s_{i}-s_{j}\right|^{2} .
$$

There are two cases to consider:

5.6. Case 1: There are $i, i^{\prime}$ so that $\gamma_{i}, \gamma_{i^{\prime}}$ are noncommuting elements of infinite order. If this happens, we will show that (A) holds; in $\$ 5.7$ we show that the complementary case leads to a contradiction.

Set

$$
w_{i}=h \exp (\underline{m}), \quad w_{i^{\prime}}=h^{\prime} \exp \left(\underline{m}^{\prime}\right)
$$

with $h, h^{\prime} \in H, \underline{m}, \underline{m}^{\prime} \in \mathfrak{m}$. Assuming (B) fails, $\|\underline{m}\|,\left\|\underline{m}^{\prime}\right\|<T^{-K_{25}}$ where $K_{25}$ is as yet undetermined.

Writing

$$
\zeta=g^{-1} h^{-1} u\left(s_{i}-s_{j}\right) g
$$

and similarly $\zeta^{\prime}$ with $i^{\prime}, h^{\prime}$ replacing $i, h$ we have that

$$
\zeta=\exp \left(g^{-1} \underline{m} g\right) \gamma_{i} \quad \zeta^{\prime}=\exp \left(g^{-1} \underline{m}^{\prime} g\right) \gamma_{i^{\prime}} .
$$

Note that since $h^{-1} u\left(s_{i}-s_{j}\right)$ preserves the form $Q_{0}=2 x z-y^{2}$, we have that $Q_{2}=Q_{0} \circ g$ is preserved by $\zeta$ as well as $\zeta^{\prime}$. We apply Lemma 5.4 on the integral system of equations in 6 variables (the coefficients of the quadratic form $Q$ )

$$
\left\{\begin{array}{l}
Q \circ \gamma_{i}=Q \\
Q \circ \gamma_{i^{\prime}}=Q
\end{array}\right.
$$

which corresponds to the matrix $A$ of the lemma and the system of equations

$$
\left\{\begin{array}{l}
Q \circ \zeta=Q \\
Q \circ \zeta^{\prime}=Q
\end{array}\right.
$$

corresponding to $B$. By (5.5c) we know that $\left\|\gamma_{i}\right\|,\left\|\gamma_{i^{\prime}}\right\|<C_{2} T^{2}$ hence

$$
\begin{aligned}
\|A-B\| & <C_{3} T^{2} T^{-K_{25}} \\
\|A\| & <C_{3} T^{4} .
\end{aligned}
$$

It is also clear that $Q_{2}$ solves the system of equations (5.6b). 
It follows from Lemma 5.4 that if

$$
\|A-B\|\|A\|^{5} \leq C_{3}^{6} T^{22-K_{25}}<\kappa_{26}
$$

there is a rational quadratic form $Q_{3}$ which is invariant under $\gamma_{i}, \gamma_{i^{\prime}}$. (2) of Lemma 5.4 implies that $Q_{3}$ is nondegenerate, hence since $\gamma_{i}$ and $\gamma_{i^{\prime}}$ do not commute Lemma 5.3 implies that the space of solutions of (5.6a) is one-dimensional. It follows from (3) of Lemma 5.4 that all coefficients of $Q_{3}$ are bounded by $\leq K_{28} C_{3} T^{20}$, hence $1 \leq\left|\operatorname{det} Q_{3}\right| \ll T^{60}$. We also note that since $g$ is in a compact region of $X$ depending only on $\delta$, the norm $\left\|Q_{0} \circ g\right\|$ can be bounded in terms of $\delta$. Thus by (2) of Lemma 5.4 and (5.6c)

$$
\left\|Q_{0} \circ g-\frac{1}{\operatorname{det} Q_{3}{ }^{1 / 3}} Q_{3}\right\| \ll T^{\left(2-K_{25}\right) / 5} .
$$

Writing $\frac{1}{\operatorname{det} Q_{3}{ }^{1 / 3}} Q_{3}=Q_{0} \circ g_{1}$ and $x_{1}=\left[g_{1}\right]_{\Gamma}$ it follows that

$$
\operatorname{disc}_{Q}\left(H \cdot x_{1}\right) \ll T^{60} \quad d\left(x, x_{1}\right) \ll T^{\left(2-K_{25}\right) / 5},
$$

establishing (A) if e.g. $K_{25}$ was chosen to be $5 M+22{ }^{(2)}$

5.7. Case 2: All $\gamma_{i}$ commute with each other. In this case $\gamma_{1}, \ldots, \gamma_{N}$ generate an abelian subgroup of $G$, and either all elements of this group are unipotent or it is contained in a $\mathbb{Q}$-torus of $\mathrm{SL}(3)$.

For any $\mathbb{Q}$-torus $L$ of $\operatorname{SL}(3, \mathbb{R})$, we have that

$$
\left|L \cap B_{T}^{G}\right| \leq C_{4} \log T^{2}
$$

with $C_{4}$ absolute, which clearly contradicts the fact that $N$, the number of the $\gamma_{i}$, is $\gg T^{1-8 \eta}$ if $T$ is large (and $\eta$ was chosen to be $<1 / 8$ ).

Therefore all the $\gamma_{i}$ are unipotent, and they all have a common fixed vector. In fact, as we have observed in the proof of Lemma 4.7, if the $K$ chosen above on p. 18 was large enough, all the $\gamma_{i}$ are generic unipotents. Let $\mathbf{n} \in \mathbb{Z}^{3}$ be a primitive vector fixed by all of the $\gamma_{i}$. This vector is already determined by $\gamma_{j+1}$; hence $\|\mathbf{n}\|$ is bounded by a power of $\left\|\gamma_{j+1}\right\|$. Indeed, e.g. using the fact that the range of $\gamma_{j+1}-\mathbf{e}$ is two-dimensional by a pigeonhole argument similar to that given that the end of the proof of Lemma 5.4 it follows that

$$
\|\mathbf{n}\| \leq C_{5}\left\|\gamma_{j+1}\right\|^{2} \leq C_{6}\left|s_{j+1}-s_{j}\right|^{4} \leq C_{7} T^{32 \eta} .
$$

For notational simplicity assume $j<N / 2$ (the other case being essentially identical). Applying both sides of $(5.5 \mathrm{~b})$ to $\mathbf{n}$ we see that for all $i$, in particular for $i=j+N / 4, j+N / 2$

$$
\left\|u\left(s_{i}-s_{j}\right) g \mathbf{n}\right\|=\left\|w_{i} g \mathbf{n}\right\| \leq 2\|g \mathbf{n}\|,
$$

\footnotetext{
${ }^{(2)}$ Here it would be sufficient to take $K_{25}=5 M+2$, but in $5.6 \mathrm{~d}$ we implicitly assumed that $K_{25}>22$.
} 
with $g \in G$ as in (5.5a). Write $g \mathbf{n}=\left(v_{1}, v_{2}, v_{3}\right)^{\top}, p(t)=v_{1}+v_{2} t+v_{3} t^{2} / 2$. A considering the first component of the vector appearing on the LHS of (5.7a), we conclude that

$$
|p(t)|<2\|g\|\|\mathbf{n}\|<C_{8} T^{32 \eta} \quad \text { for } t=0, t_{1}, t_{2}
$$

where $t_{1}=s_{j+N / 4}-s_{j}, t_{2}=s_{j+N / 2}-s_{j}$. As

$$
p(t)=p(0) \frac{\left(t-t_{1}\right)\left(t-t_{2}\right)}{t_{1} t_{2}}+p\left(t_{1}\right) \frac{t\left(t_{2}-t\right)}{t_{1}\left(t_{2}-t_{1}\right)}+p\left(t_{2}\right) \frac{t\left(t-t_{1}\right)}{t_{2}\left(t_{2}-t_{1}\right)}
$$

and using the fact that $N / 4 \leq t_{1}, t_{2}, t_{2}-t_{1} \leq T$ (where the lower bound follows from the $s_{i}$ being 1 -seperated) we conclude that

$$
\begin{aligned}
& \left|v_{1}\right| \leq C_{8} T^{32 \eta} \\
& \left|v_{2}\right| \leq 1000 C_{8} T^{1+32 \eta} N^{-2} \leq C_{9} T^{-1+48 \eta} \\
& \left|v_{2}\right| \leq 1000 C_{8} T^{32 \eta} N^{-2} \leq C_{9} T^{-2+48 \eta}
\end{aligned}
$$

Applying $a(-(1-\delta) \log T+10+\log (1 / \kappa))$ to the vector $g \mathbf{n}$, we find a vector

$$
v^{\prime} \in a(-(1-\delta) \log T+10+\log (1 / \kappa)) g \mathbb{Z}^{3}
$$

with

$$
\left\|v^{\prime}\right\|_{\infty} \leq C_{9} \kappa^{-1} \min \left(T^{-1+\delta+48 \eta}\right) .
$$

On the other hand, by construction,

$$
u\left(s_{j}\right) . x \in \Xi\left((1-\delta) \log T-\log (1 / \kappa)-10, \kappa_{23}, \delta\right),
$$

SO

$$
\left\|v^{\prime}\right\|_{\infty} \geq c_{10} \kappa_{23}\left(\kappa^{-1} T^{1-\delta}\right)^{\delta}
$$

which is a contradiction if we chose $\eta$ so that

$$
\delta(1-\delta)<1-\delta-48 \eta
$$

and $T$ is sufficiently large (depending on $\delta, \kappa, M$ and $\eta$ ).

\section{Proof of Proposition 3.7}

6.1. Using the Closing Lemma of $\$ 5$ and the nondivergence estimates of $\$ 4$ we can now finish the proof of Proposition 3.7. For the convenience of the reader, we reproduce the statement of this proposition:

6.2. Proposition (Proposition 3.7). Let $\delta, \eta, \epsilon \in(0,1)$ and $x_{1} \in X_{\eta}$. Then for any

$$
T>T_{0}(\delta, \epsilon) \eta^{-K_{8}}
$$

at least one of the following holds:

(i) There is a point $x_{2} \in G / \Gamma$ so that $H . x_{2}$ is periodic and

$$
\begin{aligned}
& \operatorname{disc}_{Q}\left(H . x_{2}\right)<T^{3 \epsilon} \\
& d_{G / \Gamma}\left(x_{1}, x_{2}\right)<T^{-1} .
\end{aligned}
$$


(ii) For any $\tau \in\left[1, T^{\epsilon \kappa 9}\right]$ there are $h, h^{\prime} \in B_{T^{K_{10}}}^{H_{10}}, x^{\prime} \in \Xi\left(\epsilon \kappa_{11} \log T, \kappa_{12}, \delta\right)$ and $\tau^{\prime} \in[\tau / 2, \tau]$ so that

$$
\begin{array}{r}
d_{G / \Gamma}\left(v(-\tau) \cdot x^{\prime}, h \cdot x_{1}\right)<c_{1} T^{-\epsilon \kappa_{13}} \\
d_{G / \Gamma}\left(v(\tau) \cdot x^{\prime}, h^{\prime} \cdot x_{1}\right)<c_{1} T^{-\epsilon \kappa_{13}}
\end{array}
$$

We recall that $K_{10}$ will be an absolute constant and $c_{1}, \kappa_{9}, \kappa_{11}, \kappa_{12}, \kappa_{13}$ are allowed to depend only on $\delta$.

Proof of Proposition 3.7. Let $\delta, \eta, \epsilon>0$ and $x_{1} \in X_{\eta}$ be given. Assume alternative (i) in the statement of Proposition 3.7 does not hold, i.e. there is no point $y \in G / \Gamma$ so that H.y is periodic and

$$
\begin{aligned}
& \operatorname{disc}_{Q}(H . y)<T^{3 \epsilon} \\
& d_{G / \Gamma}\left(x_{1}, y\right)<T^{-1} .
\end{aligned}
$$

By Proposition 4.5, for an appropriately chosen $\kappa=\kappa(\delta)>0$, there is a $t_{1} \in\left[0, K_{17}\right]$ with $x_{2}=a(\log \eta) u^{-}\left(t_{1}\right) . x_{1} \in \Xi(\infty, \kappa, \delta)$.

Now apply Lemma 5.2 on $x_{2}$ with $T_{1}=T^{\epsilon / 20}$ and $M=\frac{25}{\epsilon}$. If (A) of Lemma 5.2 holds, then there is a $H$-periodic $y$ with

$$
\begin{aligned}
& \operatorname{disc}_{Q}(H . y)<T_{1}^{60}=T^{3 \epsilon} \\
& d_{G / \Gamma}\left(x_{2}, y\right)<T_{1}^{M}=T^{-1.2} .
\end{aligned}
$$

It follows that

$$
d_{G / \Gamma}\left(x_{1}, u^{-}\left(-t_{1}\right) a(-\log \eta) . y\right) \ll \eta^{-2} T^{-1.2} .
$$

which contradicts our assumption that alternative (i) in the statement of Proposition 3.7 does not hold if $T>C_{2} \eta^{-10}$.

Thus setting $K_{8}=11$ and $T_{0}$ larger than an absolute constant we may conclude that there are $s_{2}, s_{2}^{\prime} \in\left[0, T_{1}\right], h \in H$ and $\underline{m} \in \mathfrak{m}$ so that $x_{3}=u\left(s_{2}\right) \cdot x_{2}$ and $x_{3}^{\prime}=u\left(s_{2}\right) \cdot x_{2}$ satisfy that $x_{3}=h \exp (\underline{m}) \cdot x_{3}^{\prime}$ with

$$
\begin{gathered}
\|h-e\|<T_{1}^{-\kappa_{24}}, \quad T_{1}^{-K_{25}}<\underline{m}<T_{1}^{-\kappa_{24}} \\
x_{3}, x_{3}^{\prime} \in X_{\kappa_{23}}
\end{gathered}
$$

with $K_{25}=5 M+22$ and $\kappa_{23}, \kappa_{24}$ absolute constants. Lemma 5.2 provides us with a bit more information on $x_{3}, x_{3}^{\prime}$, namely that they are in $\Xi\left(\log T_{1}, \kappa_{23}, \delta\right)$, but we shall not be using this, as we have to apply Proposition 4.5 again in any case to avoid $\underline{m}$ being very close to the Lie algebra $\mathfrak{v}$ of $V$.

Let $x_{4}=\exp (\underline{m} / 2) \cdot x_{3}^{\prime}$. Applying Proposition 4.5 we may conclude that for most $t_{2} \in\left[0, K_{17}\right]$ the point $a(r) u^{-}\left(t_{2}\right) x_{4} \in \Xi(\infty, \kappa(\delta), \delta)$ with $r=\log \left(1 / \kappa_{23}\right)$. Recalling the decomposition $\mathfrak{m}=\bigoplus_{i=-2}^{2} \mathfrak{m}_{i}$ with $\mathfrak{m}_{2}=\mathfrak{v}$ from $\$ 5.1$, the $\mathfrak{m}_{-2}$ component of $\operatorname{Ad}\left(u^{-}\left(t_{2}\right)\right) \underline{m}$ is a polynomial of degree 4 in $t_{2}$ with coefficients that are essentially given by the components of $\underline{m}$. Let $\phi_{\underline{m}}: \mathbb{R} \rightarrow \mathfrak{m}_{-2}$ denote this polynomial. Since for most $t_{2} \in\left[0, K_{17}\right]$ the norm of the polynomial $\phi\left(t_{2}\right)$ is comparable 
to the norm of its largest coefficient, we can conclude from the above that we can find a $t_{2} \in\left[0, K_{17}\right]$ so that

$$
a(r) u^{-}\left(t_{2}\right) x_{4} \in \Xi(\infty, \kappa(\delta), \delta) \text { and }\left\|\phi_{\underline{m}}\left(t_{2}\right)\right\| \geq c_{3}\|m\| .
$$

Let $x_{5}=a(r) u^{-}\left(t_{2}\right) x_{4}$ and $\underline{m}^{\prime}=\operatorname{Ad}\left(a(r) u^{-}\left(t_{2}\right)\right) \underline{m}$. In addition to being in $\Xi(\infty, \kappa(\delta), \delta)$, this point has the property that

$$
\exp \left( \pm \underline{m^{\prime}} / 2\right) \cdot x_{5} \in B_{C_{4} T_{1}}^{H} .
$$

Write $\underline{m}^{\prime}$ as $\underline{m}^{\prime}=\underline{m}_{-2}+\cdots+\underline{m}_{2}$ with $\underline{m}_{i} \in \mathfrak{m}_{i}$, and note that by choice of $t_{2}$,

$$
\left\|\underline{m}_{-2}\right\| \gg\left\|\underline{m}^{\prime}\right\| \quad T_{1}^{-K_{25}} \ll\left\|\underline{m}^{\prime}\right\| \ll T_{1}^{-\kappa_{24}} .
$$

Suppose now that $\tau \geq 1$ is given. Let $T_{2}>0$ be the smallest such that

$$
\begin{gathered}
\qquad \operatorname{Ad}\left(u\left(T_{2}\right)\right) \underline{m^{\prime}} \|=\tau \\
\text { (recall that in our normalizations } \left.\left\|\left(\begin{array}{lll}
0 & 0 & \tau \\
0 & 0 & 0 \\
0 & 0 & 0
\end{array}\right)\right\|=\tau\right) .
\end{gathered}
$$

In the degree 4 polynomial $t \mapsto \operatorname{Ad}(u(t)) \underline{m^{\prime}}$ the coefficient of the 4th order term is essentially $\underline{m}_{-2}$. As $\left\|\underline{m}_{-2}\right\| \gg T_{1}^{-K_{25}}$ we have that $T_{2} \ll T_{1}^{K_{25} / 4} \tau^{1 / 4}$ for some $C_{4}>0$; indeed, in view of the explicit form of $K_{25}$ given above $T_{2} \ll T_{1}^{20 \epsilon^{-1}+5.5} \tau^{1 / 4} \ll T^{C_{5}} \tau^{1 / 4}$ for some absolute constant $C_{5}$. As $\left\|\underline{m}^{\prime}\right\| \ll T_{1}^{-\kappa_{24}}$ it follows that $T_{2} \gg T_{1}^{c_{6}} \tau^{1 / 4}$ (with $c_{6}$ depending on $\delta$ ).

Since for an appropriate absolute constant $c_{7} \in(0,1)$ we have that

$$
\|\operatorname{Ad}(u(t)) \underline{m}\| \geq \frac{\tau}{2} \quad \text { for } t \in\left[\left(1-c_{7}\right) T_{2}, T_{2}\right] .
$$

By Proposition 4.4 it follows that there is a $t_{3} \in\left[\left(1-c_{7}\right) T_{2}, T_{2}\right]$ for which

$$
u\left(t_{3}\right) \cdot x_{5} \in \Xi\left((1-\delta) \log T_{2}-\log (1 / \kappa(\delta))-10, \tilde{\kappa}, \delta\right)
$$

provided that

$$
\frac{K_{16} T \tilde{\kappa}^{1 / 2}}{1-e^{-\delta / 2}}<c_{7}
$$

Finally, consider the polynomials $\mathbb{R} \rightarrow \mathfrak{m}$

$$
\operatorname{Ad}(t) \underline{m}_{i}
$$

for $-2 \leq i \leq 2$. In each, the highest order term is its $\mathfrak{m}_{2}$ component, and all nonzero coefficients of $t \mapsto \operatorname{Ad}(t) \underline{m}_{i}$ are of the order $\left\|\underline{m}_{i}\right\|$. It follows from definition of $T_{2}$ that for $t \in\left[0, T_{2}\right]$, the components of $\operatorname{Ad}(t) \underline{m}$ in $\mathfrak{m}_{i}$ for $i \neq 2$ are $\ll \tau T_{2}^{-1}$. Let $x_{6}=u\left(t_{3}\right) \cdot x_{5}$ and $\tau^{\prime} \geq 0$ so that the $\mathfrak{m}_{2}$ component of $\operatorname{Ad}\left(t_{3}\right) \underline{m}^{\prime}$ has $\tau^{\prime}$ in its right upper corner, i.e. is equal to $v_{\tau^{\prime}}-\mathbf{e}$. Then

$$
x_{6} \in \Xi\left((1-\delta) \log T_{2}-\log (1 / \kappa(\delta))-10, \tilde{\kappa}, \delta\right)
$$


and

$$
d\left(v\left( \pm \tau^{\prime} / 2\right) \cdot x_{6}, \exp \left( \pm\left(\operatorname{Ad}\left(t_{3}\right) \underline{m}^{\prime}\right) / 2\right) \cdot x_{6}\right) \ll \tau T_{2}^{-1} .
$$

As

$$
\exp \left( \pm\left(\operatorname{Ad}\left(t_{3}\right) \underline{m}^{\prime}\right) / 2\right) \cdot x_{6} \in B_{C_{8}\left(T_{1}+T_{2}\right)}^{H} \cdot x_{1}
$$

the proposition is proved.

\section{Divergence PRoperties of NEARBy $U$-ORBits}

7.1. In order to study the divergence properties of $u_{t}$-orbits, we make use of the representation $\rho: G \rightarrow \operatorname{Aut}(W)$ with $W=\mathbb{R}^{3} \oplus\left(\operatorname{sym}_{2}\left(\mathbb{R}^{3}\right)\right)^{*}$, which arises from the usual action of $G$ on $R^{3}$, and the action of $G$ on $\left(\operatorname{sym}_{2}\left(\mathbb{R}^{3}\right)\right)^{*}$ given after identifying $\left(\operatorname{sym}_{2}\left(\mathbb{R}^{3}\right)\right)^{*}$ with the space of ternary quadratic forms by $(g \cdot Q)(v)=Q\left(g^{-1} v\right)$.

Let $\mathbf{e}_{1}, \mathbf{e}_{2}, \mathbf{e}_{3}$ be the usual basis of $\mathbb{R}^{3}$, and $Q_{0}(x, y, z)=y^{2}-2 x z \in$ $\left(\operatorname{sym}_{2}\left(\mathbb{R}^{3}\right)\right)^{*}$. The vector $w_{0}=\mathbf{e}_{1} \oplus Q_{0} \in W$ is fixed by $U$, and an easy calculation shows that in fact $U=\operatorname{stab}_{G}\left(w_{0}\right)$. We note that the pair $\left(Q_{0}, \mathbf{e}_{1}\right)$ give a signature $(2,1)$-quadratic form, of determinant 1 , and a nonzero isotopic vector for $Q_{0}$, i.e. a nonzero vector $v$ satisfying $Q_{0}(v)=0$. Since the action of $G$ preserves these properties, it follows that $\rho(G) w_{0}$ is contained in

$$
\mathcal{G}=\{v \oplus Q: Q \text { has signature }(2,1), \operatorname{det} Q=1, v \neq 0, Q(v)=0\} .
$$

Since $G$ acts transitively on the collection of signature $(2,1)$-quadratic forms, and $H$ acts transitively on the set of nonzero isotopic vectors of $Q_{0}$ (cf. [C]) in fact $\rho(G) w_{0}=\mathcal{G}$.

7.2. We fix a rational cross section $\mathcal{W}$ to $U$ in $G$, i.e. a subvariety of $G$ so that the map $(w, u) \mapsto w u$ gives a birational map between $\mathcal{W} \times U$ and $G$. A concrete choice for $\mathcal{W}$ is

$$
\mathcal{W}=\mathcal{U}^{-} A\left(\begin{array}{lll}
1 & * & * \\
0 & 1 & 0 \\
0 & 0 & 1
\end{array}\right) .
$$

Then the map $g \mapsto \rho(g) . w_{0}$ restricted to $\mathcal{W}$ gives a birational map between $\mathcal{W}$ and the smooth quasi-affine variety $\mathcal{G}$. The rational map $\varrho: \mathcal{G} \rightarrow \mathcal{W}$ inverting this map can be given explicitly as follows: let $v_{1} \oplus Q$ be a point in $\mathcal{G}$, i.e. $Q$ is a signature $(2,1)$ quadratic form with determinant 1 and $v_{1} \in \mathbb{R}^{3} \backslash\{0\}$ with $Q\left(v_{1}\right)=0$. The quadratic form $Q(v)$ defines a bilinear form in the usual way $Q(v, w)=\frac{1}{2}(Q(v+w)-$ $Q(v)+Q(w))$. We complete $v_{1}$ to basis $v_{1}, v_{2}, v_{3}$ of $\mathbb{R}^{3}$ as follows: first find $v_{3} \in\left\langle v_{1}, \mathbf{e}_{3}\right\rangle$ satisfying

$$
Q\left(v_{1}, v_{3}\right)=-2 \quad Q\left(v_{3}\right)=0
$$

(this is possible as long as $\left.Q\left(v_{1}, \mathbf{e}_{3}\right) \neq 0\right)$. Then fix $v_{2}$ by the three linear conditions

$$
Q\left(v_{1}, v_{2}\right)=0 \quad Q\left(v_{3}, v_{2}\right)=0 \quad \operatorname{det}\left(v_{1}, v_{2}, v_{3}\right)=1 .
$$


It follows that

$$
-Q\left(v_{2}\right)=\operatorname{det}\left(Q\left(v_{i}, v_{j}\right)\right)_{i, j=1 \ldots 3}=\operatorname{det} Q \cdot \operatorname{det}\left(v_{1}, v_{2}, v_{3}\right)^{2}=-1
$$

hence if we compose $Q$ with the matrix $\left(v_{1} v_{2} v_{3}\right)$ formed by the column vectors $v_{1}, v_{2}, v_{3}$ we get $Q_{0}$. Thus $\varrho: v_{1} \oplus Q \mapsto\left(v_{1} v_{2} v_{3}\right)$ gives a rational map $\mathcal{G} \rightarrow \mathcal{W}$ inverting the map $g \mapsto g . w_{0}$; it is also clear that $\varrho$ is regular at $w_{0}$.

7.3. Lemma. Let $W_{U}$ denote the space of $\rho(U)$-invariant vectors in $W$. Then $W_{U}$ is given by

$$
W_{U}=\left\langle\mathbf{e}_{1} \oplus 0,0 \oplus Q_{0}, 0 \oplus Q_{\dagger}\right\rangle,
$$

where $Q_{0}$ is the quadratic form above and $Q_{\dagger}(x, y, z)=2 z^{2}$. The intersection $\mathcal{G} \cap W_{U}$ is a Zariski open subset of the two dimensional affine plane

$$
W_{0}=\left\{t \mathbf{e}_{1} \oplus\left(Q_{0}+t^{\prime} Q_{\dagger}\right): t, t^{\prime} \in \mathbb{R}\right\} .
$$

Proof. This is essentially a combination of the trivial observation that $\mathbf{e}_{1}$ spans the $U$-invariant vectors in $\mathbb{R}^{3}$ and the fact (easily verified by direct calculation) that any $U$-invariant ternary quadratic form is a linear combination of $Q_{0}$ and $Q_{\dagger}$ above.

Recall that $\mathcal{G}$ consists of all points $v \oplus Q$ with $v \in \mathbb{R}^{3}$ nonzero and isotropic for the signature $(2,1)$ quadratic form $Q$ of determinant 1 . If $v \oplus Q \in \mathcal{G} \cap W_{U}$ then $Q=s Q_{0}+s^{\prime} Q_{\dagger}$ and as $\operatorname{det}\left(s Q_{0}+s^{\prime} Q_{\dagger}\right)=s^{3}$ it follows that $s=1$. Moreover from the description of $\mathcal{G}$ it follows that every $v \oplus Q \in W_{0}$ with $v \neq 0$ is in $\mathcal{G}$.

7.4. One can say a bit more about $\mathcal{G} \cap W_{0}$. Our choice of cross section $\mathcal{W}$ satisfies that $A V \subset W$. Since

$$
\rho\left(\left(\begin{array}{lll}
t & & \\
& 1 & \\
& & t^{-1}
\end{array}\right) v(s)\right) w_{0}=t \mathbf{e}_{1} \oplus\left(Q_{0}+s t^{2} Q_{\dagger}\right)
$$

it follows that for $c \neq 0$

$$
\varrho\left(c \mathbf{e}_{1} \oplus\left(Q_{0}+b Q_{\dagger}\right)\right)=\left(\begin{array}{lll}
c & & \\
& 1 & \\
& & c^{-1}
\end{array}\right) v\left(\frac{b}{c^{2}}\right) .
$$

We also make note of the identity

$$
\left(\begin{array}{ccc}
c^{-1} & & \\
& 1 & \\
& & c
\end{array}\right) v(s) \varrho\left(c \mathbf{e}_{1} \oplus\left(Q_{0}+b Q_{\dagger}\right)\right)=v\left(\frac{s+b}{c^{2}}\right)
$$

that will be useful later. 
7.5. The space $W$ splits into three irreducible components under the action of $\rho(H): \mathbb{R}^{3}$, a one-dimensional representation spanned by the $\rho(H)$-fixed vector $0 \oplus Q_{0}$ and a 5 -dimensional representation (that there are only three irreducible representations follows from the fact that the space of $\rho(U)$-invariant vectors is three-dimensional).

Fix an Euclidean norm $\|\cdot\|$ on $W$ according to which these the irreducible subspaces are orthogonal and moreover in each irreducible representation of $\rho(H)$ the eigenspaces of $\rho(D)$ are orthogonal. We further scale $\|\cdot\|$ so that every $p=v \oplus Q$ with $\left\|p-w_{0}\right\| \leq 1$ satisfies that $Q$ is nondegenerate of signature $(1,2), v \neq 0$ and $\varrho$ is regular on $\mathcal{G} \cap B_{2}^{W}\left(w_{0}\right)$. For every $p \in W$ we let $\pi_{W_{U}}$ and $\pi_{W_{0}}$ denote the closest point on $W_{U}$ or $W_{0}$ to $p$ respectively, and $\pi_{W_{U}}^{\perp}(p)=p-\pi_{W_{U}}(p)$ (and similarly for $\left.W_{0}\right)$.

7.6. Lemma. For every $v \in W \backslash W_{U}$ consider the least $T \geq 0$ for which $\left\|\rho\left(u_{T}\right) v-v\right\| \geq 1$. Then

$$
\left\|\pi_{W_{U}}^{\perp} v\right\|^{-1 / 4} \ll T \ll\left\|\pi_{W_{U}}^{\perp} v\right\|^{-1}
$$

with implied constants absolute.

A similar estimate holds also for $T>0$ the least number satisfying $\|\rho(-T) v-v\| \geq 1$, or if $v \in\left(W \backslash W_{U}\right) \cap B_{1 / 2}^{W}\left(w_{0}\right)$ for $T$ the least number satisfying $\left\|\rho(-T) v-w_{0}\right\| \geq 1$.

Proof. $p(t)=\rho\left(u_{t}\right) v-v$ is a vector valued polynomial of degree $\leq 4$ whose maximal nonconstant coefficient is bounded above and below by absolute constants times $\left\|\pi_{W_{U}}^{\perp} v\right\|$. In particular, $p(t)$ is not a constant as $v \notin W_{U}$, hence $\|p(t)\| \rightarrow \infty$ as $t \rightarrow \infty$. The estimate (7.6a) for $T=\min \{t \geq 0:\|p(t)\| \geq 1\}$ or $T=\min \{t \geq 0:\|p(-t)\| \geq 1\}$ now follows from general properties of degree $\leq 4$ polynomials. The case of $T=\min \left\{t \geq 0:\left\|\rho\left(u_{t}\right) v-w_{0}\right\| \geq 1\right\}$ follows similarly by taking $p_{1}(t)=\rho\left(u_{t}\right) v-w_{0}$, and noting that by construction $\left\|p_{1}(0)\right\| \leq 1 / 2$ while $\left\|p_{1}(T)\right\|=1$.

7.7. Lemma. Let $v \in\left(W \backslash W_{U}\right) \cap B_{1 / 2}^{W}\left(w_{0}\right)$ and

$$
T=\min \left\{t \geq 0:\left\|\rho\left(u_{t}\right) v-w_{0}\right\| \geq 1\right\} .
$$

Then for $0 \leq t \leq T$ it holds that

$$
\left\|\pi_{W_{U}}^{\perp}\left(\rho\left(u_{t}\right) v\right)\right\| \ll T^{-1} \text {. }
$$

Proof. Suppose $t \leq T / 2$. Write $v^{\prime}=\frac{1}{2} \rho\left(u_{t}\right) v$, and apply Lemma 7.6 to $v^{\prime}$. Since $\left\|\rho\left(u_{s}\right) v-w_{0}\right\|<1$ for all $s \in[0, T]$, it follows that for $s \in[0, T / 2]$

$$
\left\|\rho\left(u_{t+s}\right) v-\rho\left(u_{t}\right) v\right\| \leq 2 \quad \text { for } 0 \leq s \leq T / 2 .
$$

Consequently, $v^{\prime}$ satisfies the conditions of Lemma 7.6 for $T^{\prime} \geq T / 2$. Thus by the second inequality in (7.6a)

$$
T \leq 2 T^{\prime} \ll\left\|\pi_{W_{U}}^{\perp}\left(v^{\prime}\right)\right\|^{-1},
$$


which is equivalent to $7.7 \mathrm{a}$.

7.8. Lemma. Let $g \in G$ satisfies that $\rho(g) w_{0} \in B_{1}^{W}\left(w_{0}\right)$. Then

$$
\left\|\pi_{W_{0}}^{\perp}\left(\rho(g) w_{0}\right)\right\| \gg \ll\left\|\pi_{W_{U}}^{\perp}\left(\rho(g) w_{0}\right)\right\| .
$$

Proof. Since $W_{0}$ is an affine subspace of $W_{U}$ the left-hand side of (7.8a) is $\geq$ the right hand side. To see the opposite inequality, let $\pi_{W_{U}}\left(\rho(g) w_{0}\right)=a \mathbf{e}_{1} \oplus\left(a_{1} Q_{0}+a_{2} Q_{\dagger}\right)$ with $Q_{0}, Q_{\dagger}$ as in Lemma 7.3 and $a, a_{1}, a_{2} \in \mathbb{R}$. Since $\rho(g) w_{0} \in \mathcal{G}$, it has the form $v \oplus Q$ with $Q$ an indefinite quadratic form of determinant 1 . As $\operatorname{det}\left(a_{1} Q_{0}+a_{2} Q_{\dagger}\right)=a_{1}^{3}$, and since by the conditions on $g$, the scalar $a_{1}$ is in some fixed compact subset of $(0, \infty)$,

$$
\left|a_{1}-1\right| \ll\left\|\rho(g) w_{0}-\pi_{W_{U}}\left(\rho(g) w_{0}\right)\right\|=\left\|\pi_{W_{U}}^{\perp}\left(\rho(g) w_{0}\right)\right\| .
$$

But this in turn implies that

$$
\left\|\rho(g) w_{0}-\frac{a}{a_{1}} \mathbf{e}_{1} \oplus\left(Q_{0}+\frac{a_{2}}{a_{1}} Q_{\dagger}\right)\right\| \ll\left\|\pi_{W_{U}}^{\perp}\left(\rho(g) w_{0}\right)\right\| .
$$

On the other hand, as $\frac{a}{a_{1}} \mathbf{e}_{1} \oplus\left(Q_{0}+\frac{a_{2}}{a_{1}} Q_{\dagger}\right) \in W_{0}$ the left-hand side of the above equation is $\geq\left\|\pi_{W_{0}}^{\perp}\left(\rho(g) w_{0}\right)\right\|$ which concludes the proof.

7.9. Let $\mathbf{u}=\left(\begin{array}{lll}0 & 1 & \\ & 0 & 1 \\ & & 0\end{array}\right)$ so that $U=\exp (\mathbb{R} \mathbf{u})$. The following is a consequence of the elementary theory of finite dimensional representations of $\operatorname{sl}(2, \mathbb{R}) \cong$ Lie $H$ :

7.10. Lemma. Let $d \rho$ denote the representation of $\mathfrak{g}$ corresponding to $\rho$. Then for every $\underline{n}$ it holds that

$$
\left\|\pi_{W_{U}}^{\perp}\left(d \rho(\underline{n}) w_{0}\right)\right\| \gg\left\|\underline{u}_{-}\right\|
$$

where $\underline{n}=\underline{u}_{-}+\underline{a}+\underline{u}_{+}$with $\underline{u}_{ \pm} \in \operatorname{Lie}\left(\mathcal{U}^{ \pm}\right)$and $\underline{a} \in \operatorname{Lie} A$

Proof. Decompose $[\mathbf{u}, \underline{n}]$ also as $[\mathbf{u}, \underline{n}]=\underline{u}_{-}^{\prime}+\underline{a}^{\prime}+\underline{u}_{+}^{\prime}$. Note that as

$$
\mathbf{u}, \mathbf{d}=\left(\begin{array}{ccc}
1 & & \\
& 0 & \\
& & -1
\end{array}\right),\left(\begin{array}{lll}
0 & & \\
1 & 0 & \\
& 1 & 0
\end{array}\right)
$$

form a $\operatorname{sl}(2, \mathbb{R})$-triplet, $\left\|\underline{u}_{-}^{\prime}+\underline{a}^{\prime}\right\| \gg\left\|\underline{u}_{-}\right\|$.

Since $d \rho$ is a Lie algebra representation and $d \rho(\mathbf{u}) w_{0}=0$,

$$
d \rho([\mathbf{u}, \underline{n}]) w_{0}=(d \rho(\mathbf{u}) d \rho(\underline{n})-d \rho(\underline{n}) d \rho(\mathbf{u})) w_{0}=d \rho(\mathbf{u}) d \rho(\underline{n}) w_{0} .
$$

As $W_{U}$ is by definition the kernel of $d \rho(\mathbf{u})$, it follows that

$$
\left\|\pi_{W_{U}}^{\perp} d \rho(\underline{n}) w_{0}\right\| \gg\left\|d \rho([\mathbf{u}, \underline{n}]) w_{0}\right\| .
$$

Decompose the space $W$ into $d \rho(\mathbf{d})$ eigenspaces. The vector $w_{0}$ is in such an eigenspace, with eigenvalue one. Hence $d \rho\left(\underline{u}_{-}^{\prime}+\underline{a}^{\prime}\right) w_{0}$ has components in the eigenspaces with eigenvalue $\leq 1$, and $d \rho\left(\underline{u}_{+}^{\prime}\right) w_{0}$ in 
eigenspaces with eigenvalue $>1$. By our choice of norm on $W$ it follows that

$$
\left\|d \rho([\mathbf{u}, \underline{n}]) w_{0}\right\| \geq\left\|d \rho\left(\underline{u}_{-}^{\prime}+\underline{a}^{\prime}\right) w_{0}\right\| \gg\left\|\underline{u}_{-}^{\prime}+\underline{a}^{\prime}\right\| \gg\left\|\underline{u}_{-}\right\|,
$$

hence by $7.10 \mathrm{a}),\left\|\pi_{W_{U}}^{\perp} d \rho(\underline{n}) w_{0}\right\| \gg\left\|\underline{u}_{-}\right\|$.

7.11. Proposition. Let $h_{0}=u_{-} a u_{+} \in B_{\kappa_{29}}^{G} \backslash N_{G}(U)$ with $u_{-} \in \mathcal{U}^{-}, u_{+} \in$ $\mathcal{U}^{+}, a \in A$ and $\kappa_{29}$ a sufficiently small absolute constant. Then there are

(i) $T>0$ with $\left\|h_{0}-\mathbf{e}\right\|^{-1 / 4} \ll T \ll\left\|u_{-}-\mathbf{e}\right\|^{-1}$,

(ii) polynomials $c(t), b(t)$ with $F_{t}(x)=c(t)^{-2}(x+b(t))$ satisfying

$$
\begin{aligned}
\max _{x \in\left[-\frac{1}{2}, \frac{1}{2}\right]}\left|F_{t}(x)-x\right| & \leq \kappa_{30} \quad \text { for } t \in[-T, T], \\
& =\kappa_{30} \quad \text { for } t=-T \text { or } T
\end{aligned}
$$

(iii) a rational function $\phi:[-T, T] \rightarrow \mathbb{R}$ satisfying

$$
K_{31}^{-1}\left|t_{1}-t_{2}\right| \leq\left|\phi\left(t_{1}\right)-\phi\left(t_{2}\right)\right| \leq K_{31}\left|t_{1}-t_{2}\right| \quad \text { for all } t_{1}, t_{2} \in[-T, T]
$$

so that for every $t \in[-T, T]$ and $p \in G / \Gamma$,

$$
d\left(u(t) h_{0} \cdot p,\left(\begin{array}{ccc}
c(t) & & \\
& 1 & \\
& & c(t)^{-1}
\end{array}\right) v\left(\frac{b(t)}{c(t)^{2}}\right) u(\phi(t)) \cdot p\right) \ll T^{-1} .
$$

Note that it follows from the above equation (or from the definition of $\phi$ below) that $\phi(0)$ is small; e.g. a suitable choice of $\kappa_{29}$ would guarantee that $|\phi(0)|<1$.

Proof of Proposition 7.11. Let $T_{1}=\min \left\{t \geq 0:\left\|\rho\left(u_{t} h_{0}\right) w_{0}-w_{0}\right\| \geq\right.$ $1\}$. By Lemma 7.3, we can write the polynomial map $t \mapsto \pi_{W_{0}}\left(\rho\left(u_{t} h_{0}\right) w_{0}\right)$ as

$$
\pi_{W_{0}}\left(\rho\left(u_{t} h_{0}\right) w_{0}\right)=c(t) \mathbf{e}_{1} \oplus\left(Q_{0}+b(t) Q_{\dagger}\right)
$$

with $c(t), b(t)$ real valued polynomials of degree $\leq 4$.

Define $\phi(t)$ by

$$
\varrho\left(\rho\left(u_{t} h_{0}\right) w_{0}\right)=u_{t} h_{0} u_{-\phi(t)},
$$

i.e. $u_{\phi(t)}=\left(\varrho\left(\rho\left(u_{t} h_{0}\right) w_{0}\right)\right)^{-1} u_{t} h_{0}$. Note that such $\phi(t)$ must exist as $\varrho\left(\rho(g) w_{0}\right) \in g U$ for every $g \in G$. Since $\varrho$ is a rational function on $\mathcal{G}$ and is well behaved on $\mathcal{G} \cap B_{2}^{W}\left(w_{0}\right)$, the function $\phi$ defined above is rational, and well behaved as a function of $t$ as long as $\rho\left(u_{t} h_{0}\right) w_{0}$ remains in $\overline{B_{1}^{W}\left(w_{0}\right)}$.

By Lemma 7.7 and 7.8 it follows that for $|t| \leq T_{1}$

$$
\left\|\pi_{W_{0}}^{\perp}\left(\rho\left(u_{t} h_{0}\right) w_{0}\right)\right\| \ll T_{1}^{-1}
$$


hence by the regularity of $\varrho$ on $\mathcal{G} \cap \overline{B_{1}^{W}\left(w_{0}\right)}$

$$
\begin{aligned}
d_{G}\left(\varrho\left(\rho\left(u_{t} h_{0}\right) w_{0}\right), \varrho\left(\pi_{W_{0}}\left(\rho\left(u_{t} h_{0}\right) w_{0}\right)\right)\right) & = \\
d_{G}\left(u_{t} h_{0} u_{-\phi(t)}, h_{1}(t)\right) & \ll T_{1}^{-1},
\end{aligned}
$$

with

$$
h_{1}(t)=\left(\begin{array}{ccc}
c(t) & & \\
& 1 & \\
& & c(t)^{-1}
\end{array}\right) v\left(\frac{b(t)}{c(t)^{2}}\right) .
$$

Applying both of these elements to the point $u_{\phi(t)} p$ we see that

$$
d\left(u_{t} h_{0} \cdot p, h_{1}(t) u_{\phi(t)} \cdot p\right) \ll T_{1}^{-1} .
$$

If one chooses $\kappa_{30}$ appropriately, it is clear that there will be a $T_{1}$ with $T \ll T_{1}<T$ for which condition (ii) is satisfied.

Finally, regarding (iii), observes that by (7.11a) for $t_{1}, t_{2} \in[-T, T]$,

$$
u_{\phi\left(t_{1}\right)-\phi\left(t_{2}\right)}=\left(\varrho\left(\rho\left(u_{t_{1}} h_{0}\right) w_{0}\right)\right)^{-1} u_{t_{1}-t_{2}} \varrho\left(\rho\left(u_{t_{2}} h_{0}\right) w_{0}\right) .
$$

As $\varrho\left(\rho\left(u_{t} h_{0}\right) w_{0}\right)$ is in a fixed compact neighborhood of $\mathbf{e} \in G$, say $\Omega$, for all $t \in[-T, T]$, we see that for any $t_{1}, t_{2} \in[-T, T]$

$$
u_{\phi\left(t_{1}\right)-\phi\left(t_{2}\right)} \in \Omega^{-1} u_{t_{1}-t_{2}} \Omega
$$

and (iii) follows by comparing norms.

\section{Some Properties of RAtional FUnCtions}

8.1. Lemma. Let $a(t), b(t), c(t)$ be polynomials of degree $\leq d$, with $c(t)>0$ on $t \in[0,1]$. Set $F_{t}(x)=\frac{a(t)}{c(t)^{A}} x+\frac{b(t)}{c(t)^{B}}$ with $A, B$ nonnegative integers. Assume that $\left|F_{t}(x)-x\right| \leq \rho$ for all $t \in[0,1]$. Let $C=\max (A, B), M=\max _{t \in[0,1]} c(t), m=\min _{t \in[0,1]} c(t)$. Then

$$
\max _{t \in[0,1]}\left|\frac{\partial}{\partial t} F_{t}(x)\right| \leq K_{32} \rho\left(\frac{M}{m^{2}}+\left(\frac{M}{m}\right)^{C}\right) .
$$

with $K_{32}$ depending only on $d, A, B$.

Proof. Since $\left|F_{t}(x)-x\right| \leq \rho$ on $t \in[0,1]$ it follows that the coefficients of the polynomial $p(t)=c(t)^{C}\left(\frac{a(t)}{c(t)^{A}} x+\frac{b(t)}{c(t)^{B}}-x\right)$ are all $\ll M^{C} \rho$ hence $\max _{t \in[0,1]}\left|p^{\prime}(t)\right|<C_{1} M^{C} \rho$. It follows that

$$
\begin{aligned}
\left|\frac{\partial F_{t}(x)}{\partial t}\right| & \leq\left|\frac{p^{\prime}(t)}{c(t)^{C}}\right|+C\left|\frac{c^{\prime}(t)\left(F_{t}(x)-x\right)}{c(t)^{2}}\right| \\
& \leq C_{1} \rho\left(\frac{M}{m}\right)^{C}+C \rho \frac{M}{m^{2}} .
\end{aligned}
$$


8.2. Corollary. Let $c(t), b(t)$ be polynomials of degree $\leq d$, with $c(t) \in$ $\left[\delta, \delta^{-1}\right]$ on $t \in[0,1]$. Set $F_{t}(x)=c(t)^{B} x+b(t) c(t)^{A}$, with $A, B \in \mathbb{Z}$, and assume that $\left|F_{t}(x)-x\right| \leq \rho$ for all $t \in[0,1]$. Then

$$
\begin{aligned}
\max _{t \in[0,1]}\left|\frac{\partial}{\partial t} F_{t}(x)\right| & \leq K_{33} \rho \\
\max _{t \in[0,1]}\left|\frac{\partial}{\partial t} F_{t}^{-1}(x)\right| & \leq K_{33} \rho .
\end{aligned}
$$

with $K_{33}$ depending on $d, \delta, A, B$.

Proof. This follows directly from Lemma 8.1 as $F_{t}^{-1}(x)=x c(t)^{-A}-$ $b(t) c(t)^{B-A}$, hence that lemma applies to both $F_{t}$ and $F_{t}^{-1}$.

\section{The COMBinATORIAL LEMma}

9.1. Definition. Let $S \subset \mathbb{R}$ and I an interval. The total density of $S$ on $I$ is defined to be

$$
\delta(S ; I)=|I|^{-1} \int_{I} d_{\mathbb{R}}(x, S) d x .
$$

9.2. Lemma. Let $S$ be a closed subset of $\mathbb{R}$, and $I$ be an interval containing at least one point of $S$. Then for all $\alpha<1$

$$
m(\{x \in I: d(x, S)<\alpha \delta(S ; I)\}) \geq \alpha|I| / 8 .
$$

Proof. We first show that there is a subset $D \subset I$ of measure $\geq \alpha|I| / 4$ so that

$$
\frac{1}{m(D)} \int_{D} d(x, S) d x \leq \alpha \delta(S ; I) / 2 .
$$

Indeed, let $J$ be one of the the connected components of $I \backslash S$. Then at least one of the endpoints of $J$ is in $S$,

$$
|J| / 4 \leq|J|^{-1} \int_{J} d(x, S) d x \leq|J| / 2
$$

and therefore there is a subinterval $J^{\prime} \subset J$ with $\left|J^{\prime}\right|>\alpha|J| / 4$ so that

$$
\frac{1}{|J|^{\prime}} \int_{J^{\prime}} d(x, S) d x \leq \frac{\alpha}{2|J|} \int_{J} d(x, S) d x
$$

Taking $D$ to be the union of these subintervals $J^{\prime}$ gives (9.2a).

Now by Chebyshev, the measure of those $x \in D$ with $d(x, S)>$ $\alpha \delta(S ; I)$ is $\leq m(D) / 2$, hence there is a subset of $I$ of measure $\geq m(D) / 2$ on which $d(x, S) \leq \alpha \delta(S ; I)$ and the lemma follows.

9.3. Theorem ("Combinatorial Lemma"). Let $c(t), b(t)$ be polynomials of degree $d$ with $c(0)=1$ and $b(0)=0$ and $A, B \in \mathbb{Z}$. Set $F_{t}(x)=$ $c(t)^{A} x+b(t) / c(t)^{B}$. Assume that for $\Delta \in(0,1 / 3]$

(i) $\quad\left|F_{t}(x)-x\right| \leq \Delta$ for all $t \in[0,1]$ and $x \in[0,1]$

(ii) $\left|F_{1}(x)-x\right|=\Delta$ for some $x \in[0,1]$. 
Then there are $\kappa_{34}, \kappa_{35}>0$ depending only on $d, A, B, \Delta$ so that for every $S \subset[0,1]$ containing the endpoints of this interval the set

$$
\mathcal{G}=\left\{t \in[0,1]: \min _{\epsilon= \pm 1} \delta\left(S \cup\left[F_{t}\right]^{\epsilon}(S) ;[0,1]\right)<\frac{\delta(S ;[0,1])}{1+\kappa_{34}}\right\}
$$

satisfies that $m(\mathcal{G}) \geq \kappa_{35}$.

Proof. For any $x \in[0,1]$, let

$$
r_{x}=\max \left\{\left|F_{t}(x)-x\right|: 0 \leq t \leq 1\right\} .
$$

It follows from (i) of the statement of the theorem that

(9.3a) $c(t)^{A} \in[1-2 \Delta, 1+2 \Delta], \quad \frac{b(t)}{c(t)^{B}} \in[-\Delta, \Delta] \quad$ for all $t \in[0,1]$;

also note that for any $t \in[0,1]$

$$
\left(F_{t}\right)^{-1}(x)-x=-\left(F_{t}(x)-x\right) / c(t) .
$$

Suppose first that there is some connected component $(\alpha, \beta)$ of $[0,1] \backslash$ $S$ with $\beta-\alpha>\Delta / 40$. It follows from (ii) that ${ }^{(3)}$

$$
\max \left(\left|F_{1}(\alpha)-\alpha\right|,\left|F_{1}(\beta)-\beta\right|\right) \geq \frac{\Delta^{2}}{40}
$$

Without loss of generality, assume $\left|F_{1}(\alpha)-\alpha\right| \geq \Delta^{2} / 40$. Let $\epsilon=1$ if $F_{1}(\alpha)>\alpha$ and $\epsilon=-1$ otherwise; by $(9.3 \mathrm{a}$ ) and $(9.3 \mathrm{~b})$ it follows that

$$
F_{1}^{\epsilon}(\alpha) \geq \alpha+\frac{\Delta^{2}}{120}
$$

From Corollary 8.2 it follows that there is a set $E \subset[0,1]$ of measure $\geq c_{1}$ (depending only on $d, A$ ) so that for $t \in E$

$$
F_{t}^{\epsilon}(\alpha) \in\left(\alpha+\Delta^{2} / 240, \alpha+\Delta^{2} / 120\right)
$$

and since $\beta-\alpha \geq \Delta^{2} / 40$ it follows that for $t \in E$

$$
\delta\left(S \cup F_{t}^{\epsilon}(S) ;[0,1]\right) \leq\left(1-c_{2}\right) \delta(S ;[0,1])
$$

for $c_{2}>0$ some function of $\Delta$, and the theorem follows.

Thus we may assume for the rest of the proof that every connected component of $[0,1] \backslash S$ has size $\leq \Delta / 40$. Clearly it suffice to show that

$$
\begin{array}{r}
\int_{0}^{1} \int_{0}^{1} d\left(x, S \cup F_{t}(S)\right) d t d x+\int_{0}^{1} \int_{0}^{1} d\left(x, S \cup F_{t}^{-1}(S)\right) d t d x \\
<\left(2-c_{3}\right) \delta(S ;[0,1]),
\end{array}
$$

with $c_{3}$ depending only on $d, A, B, \Delta$.

Equations (9.3a) and $9.3 \mathrm{~b}$ imply that for any $x \in[0,1]$

$$
\left[x-r_{x} / 3, x+r_{x} / 3\right] \subset\left\{F_{t}^{\epsilon}[x]: t \in[0,1], \epsilon= \pm 1\right\} \subset\left[x-3 r_{x}, x+3 r_{x}\right] .
$$

\footnotetext{
${ }^{(3)}$ The exact value of the constant on the right hand side is irrelevant, hence we omit the details.
} 
Using the above bounds on $c(t)$ it also follows that for any $y, t \in[0,1]$

$$
\left|F_{t}(y)-F_{t}(x)\right| \leq \frac{5}{3}|y-x| \quad\left|F_{t}^{-1}(y)-F_{t}^{-1}(x)\right| \leq 3|y-x|
$$

hence for every $y \in\left[x-r_{x} / 20, x+r_{x} / 20\right]$ it holds that (9.3e)

$\left[x-r_{x} / 20, x+r_{x} / 20\right] \subset\left\{F_{t}^{\epsilon}[y]: t \in[0,1], \epsilon= \pm 1\right\} \subset\left[x-4 r_{x}, x+4 r_{x}\right]$.

Out of the intervals $\left[x-r_{x} / 20, x+r_{x} / 20\right] \cap[0,1]$ we can extract two disjoint subcollection $\mathcal{J}_{i}$ so that

$$
\bigcup_{i=1,2} \bigcup_{J \in \mathcal{J}_{i}} J=[0,1]
$$

For any connected component $I=(\alpha, \beta)$ of $[0,1] \backslash S$ we mark one endpoint as follows: Let

$$
\Phi(x)= \begin{cases}F_{1}(x) & \text { if } c(1) \leq 1 \\ F_{1}^{-1}(x) & \text { otherwise }\end{cases}
$$

Mark $\alpha$ if $\Phi$ moves it to the right more than it moves $\beta$ to the left, i.e. if

$$
\Phi(\alpha)-\alpha \geq \beta-\Phi(\beta)
$$

otherwise we mark the point $\beta$. Note that since $\Phi$ is a contraction, at least one of $\Phi(\alpha)-\alpha, \beta-\Phi(\beta)$ must be positive.

We then remove from both $\mathcal{J}_{1}$ and $\mathcal{J}_{2}$ all those intervals fully contained in $I$ but which do not intersect the third of $I$ nearest the marked endpoint of $I$; after performing this operation for every connected component of $[0,1] \backslash S$ we obtain two new disjoint collections $\tilde{\mathcal{J}}_{1}, \tilde{\mathcal{J}}_{2}$ so that for every connected component $I$ of $[0,1] \backslash S$ at least one third of $I$ is contained in the union $\bigcup_{i=1,2} \bigcup_{J \in \tilde{\mathcal{J}}_{i}} J$. It follows that

$$
\sum_{J \in \tilde{\mathcal{J}}_{1} \cup \tilde{\mathcal{J}}_{2}} \int_{J} d(x, S) d x \geq \frac{2 \delta(S ;[0,1])}{9}
$$

Hence for an appropriate choice of $i, \mathcal{J}=\tilde{\mathcal{J}}_{i}$ is a disjoint collection of intervals with

$$
\sum_{J \in \mathcal{J}} \int_{J} d(x, S) d x \geq \frac{\delta(S ;[0,1])}{9} .
$$

In order to prove (9.3c), it is clearly enough to show that for every $J \in \mathcal{J}$

$$
\begin{aligned}
& \int_{J} d x \int_{0}^{1} d\left(x, S \cup F_{t}(S)\right) d t+ \\
& \quad+\int_{J} d x \int_{0}^{1} d\left(x, S \cup F_{t}^{-1}(S)\right) d t<\left(2-\kappa_{36}\right) \int_{J} d(x, S) d x
\end{aligned}
$$

There are two cases to consider: 
The interval $J$ intersects $S$ : In this case, we apply Lemma 9.2 on $J$ to find a subset $E \subset J$ with $m(E) \geq c_{4}|J|$ so that

$$
d(y, S)<0.01 \delta(S ; J) \quad \text { for every } y \in E
$$

where $c_{4}$ is an absolute constant. Write $J=\left[x-r_{x} / 20, x+r_{x} / 20\right] \cap[0,1]$ for some $x \in[0,1]$. We recall $9.3 \mathrm{e}$, which states that for every $y \in J$ $\left[x-r_{x} / 20, x+r_{x} / 20\right] \subset\left\{F_{t}^{\epsilon}(y): t \in[0,1], \epsilon= \pm 1\right\} \subset\left[x-4 r_{x}, x+4 r_{x}\right]$; moreover, at least one of the intervals $\left[x-r_{x} / 20, y\right]$ or $\left[y, x+r_{x} / 20\right]$ is in $\left\{F_{t}(y): t \in[0,1]\right\}$, with the other interval being in $\left\{F_{t}^{-1}(y): t \in[0,1]\right\}$. For notational convenience we assume $\left[x-r_{x} / 20, y\right]$ is in that set (the other case being identical). Using Corollary 8.2 we deduce that $\left|\frac{\partial}{\partial t} F_{t}(y)\right|,\left|\frac{\partial}{\partial t} F_{t}^{-1}(y)\right|<C_{5}|J|$ for all $t \in[0,1]$. It follows that

$$
\begin{aligned}
m\left(\left\{t \in[0,1]: F_{t}(y) \in E\right\}\right) & \geq \frac{m\left(E \cap\left[x-r_{x} / 20, y\right]\right)}{\max _{t \in[0,1]}\left|\frac{\partial}{\partial t} F_{t}(y)\right|} \\
& \geq \frac{m\left(E \cap\left[x-r_{x} / 20, y\right]\right)}{C_{5}|J|} \\
m\left(\left\{t \in[0,1]: F_{t}^{-1}(y) \in E\right\}\right) & \geq \frac{m\left(E \cap\left[y, x+r_{x} / 20\right]\right)}{\max _{t \in[0,1]}\left|\frac{\partial}{\partial t} F_{t}^{-1}(y)\right|} \\
& \geq \frac{m\left(E \cap\left[y, x+r_{x} / 20\right]\right)}{2 C_{5}|J|} .
\end{aligned}
$$

We obtain that there is a choice of $\epsilon= \pm 1$ for which

$$
m\left(\left\{t \in[0,1]: F_{t}^{\epsilon}(y) \in E\right\}\right) \geq \frac{m(E)}{2 C_{5}|J|}=c_{6} ;
$$

note that $c_{6}$ depends only on $d, A, B$ and is clearly $<1$. Whenever $F_{t}^{\epsilon}(y) \in E$ it holds that

$$
d\left(y, F_{t}^{-\epsilon} S\right) \leq 3 d\left(F_{t}^{\epsilon}(y), S\right) \leq 0.03 \delta(S ; J) .
$$

This implies that

$$
\begin{array}{rl}
\int_{0}^{1} d\left(y, S \cup F_{t} S\right) d t+\int_{0}^{1} & d\left(y, S \cup F_{t}^{-1} S\right) d t \\
& \leq\left(2-c_{6}\right) d(y, S)+0.03 c_{6} \delta(S ; J) .
\end{array}
$$

Trivially, the left-hand side of $(9.3 \mathrm{~g})$ is also $\leq 2 d(y, S)$. Let $E^{\prime}=$ $\{y \in J: d(y, S) \leq 0.03 \delta(S ; J)\}$. Then

$$
\begin{aligned}
\int_{J \backslash E^{\prime}} d x \int_{0}^{1} d\left(x, S \cup F_{t}[S]\right) d t+\int_{J \backslash E^{\prime}} d x \int_{0}^{1} d\left(x, S \cup F_{t}^{-1}[S]\right) d t \\
<\left(2-c_{6}\right) \int_{J \backslash E^{\prime}} d(x, S) d x+0.03 c_{6} m\left(J \backslash E^{\prime}\right) \delta(S ; J),
\end{aligned}
$$


and clearly

$$
\begin{aligned}
2 \int_{E^{\prime}} d(x, S) d x & \leq 0.06 m\left(E^{\prime}\right) \delta(S ; J) \\
& \leq\left(2-c_{6}\right) \int_{E^{\prime}} d(x, S) d x+0.03 c_{6} m\left(E^{\prime}\right) \delta(S ; J) .
\end{aligned}
$$

Hence

$$
\begin{aligned}
\int_{J} d x \int_{0}^{1} d\left(x, S \cup F_{t}[S]\right) d t & +\int_{J} d x \int_{0}^{1} d\left(x, S \cup F_{t}^{-1}[S]\right) d t \\
& <\left(2-c_{6}\right) \int_{J} d(x, S) d x+0.03 \delta(S ; J)
\end{aligned}
$$

and (9.3f) follows, in the case $J \cap S \neq \emptyset$.

The interval $J$ is disjoint from $S$ : Again, write $J=\left[x-r_{x} / 20, x+\right.$ $\left.r_{x} / 20\right] \cap[0,1]$ for some $x \in[0,1]$, and let $I=(\alpha, \beta)$ be the connected component of $[0,1] \backslash S$ containing $J$. Set $\ell=(\beta-\alpha)$. Since $S$ intersects every interval of size $\frac{\Delta}{40}$, it follows that $r_{x}<\Delta / 2$ and hence in particular $|(c(1)-1) x+b(1)|<\Delta / 2$. Condition (ii) of the statement of the theorem implies that there is some $x^{\prime} \in[0,1]$ for which $\left|(c(1)-1) x^{\prime}+b(1)\right|=\Delta$, hence $|c(1)-1| \geq \frac{\Delta}{2}$. Without loss of generality, we may assume that in the terminology introduced on p. 32 the point $\alpha$ is the marked endpoint of the interval $(\alpha, \beta)$.

Let $\Phi_{t}=\left(F_{t}\right)^{\epsilon}$ where $\epsilon=1$ if $c(1) \leq 1$ and $\epsilon=-1$ otherwise. Then

$$
\frac{\partial}{\partial y} \Phi_{t}(y) \leq \frac{2}{2+\Delta}
$$

and hence

$$
\left(\Phi_{1}(\alpha)-\alpha\right)+\left(\beta-\Phi_{1}(\beta)\right) \geq \frac{\Delta \ell}{2+\Delta} .
$$

Since $\alpha$ is marked, we have that $\Phi_{1}(\alpha)-\alpha \geq\left(\beta-\Phi_{1}(\beta)\right.$ hence

$$
\Phi_{1}(\alpha)>\alpha+\frac{\Delta \ell}{5} .
$$

Also, by definition of $r_{x}$ and $\left.9.3 \mathrm{a}\right)$, for all $t \in[0,1]$

$$
\left|\Phi_{t}(x)-x\right|<3 r_{x}
$$

and it follows from $9.3 \mathrm{~d}$ that for all $t \in[0,1]$

$$
\left|\Phi_{t}(\alpha)-\alpha\right|<3 r_{x}+3(x-\alpha)<6(x-\alpha) .
$$

In particular, setting $t=1$ and comparing with $9.3 \mathrm{~h}$ we see that $x>\alpha+\frac{\Delta \ell}{30}$. Since

$$
\int_{J} d(x, S) d x=\int_{J \cap\left[\alpha, \alpha+\frac{\ell}{2}\right]}|x-\alpha| d x+\int_{J \cap\left[\alpha+\frac{\ell}{2}, \beta\right]}|\beta-x| d x
$$


and since by assumption $J \cap[\alpha, \alpha+\ell / 3] \neq \emptyset$ it follows that

$$
\int_{J \cap\left[\alpha+\frac{\ell}{200}, \alpha+\frac{\ell}{2}\right]}|x-\alpha| d x \geq c_{7} \int_{J} d(x, S) d x .
$$

Now by Corollary $8.2,(9.3 \mathrm{~h})$, and (9.3i) there is some $c_{8}$ depending only on the $d, A, B, \Delta$ so that if

$$
E_{J}=\left\{t \in[0,1]: \frac{x-\alpha}{200}<\Phi_{t}[\alpha]-\alpha<\frac{x-\alpha}{100}\right\}
$$

then $m\left(E_{J}\right)>c_{8}$. If $t \in E_{J}$

$$
\int_{J \cap\left[\alpha+\frac{\ell}{200}, \alpha+\frac{\ell}{2}\right]}\left|\left(\Phi_{t}(\alpha)\right)^{\epsilon}[\alpha]-x\right| d x \leq 0.99 \int_{J \cap\left[\alpha+\frac{\ell}{200}, \alpha+\frac{\ell}{2}\right]}|x-\alpha| d x
$$

and so by $9.3 \mathrm{j})$

$$
\int_{J} d\left(x, S \cap \Phi_{t} S\right) d x \leq\left(1-0.01 c_{7}\right) \int_{J} d(x, S) d x
$$

and

$$
\int_{0}^{1} \int_{J} d\left(x, S \cap \Phi_{t} S\right) d x d t \leq\left(1-0.01 c_{7} c_{8}\right) \int_{J} d(x, S) d x
$$

\section{The MAIN LEMMA}

We now state our main "bootstrapping" lemma, which we derive from combining the combinatorial lemma of the previous section (Theorem 9.3 with the properties of the $U$-action developed in sections $\$ 4$ and $\$ 7$.

10.1. Main Lemma. Let $\delta, \kappa \in(0,1)$, and $k \geq k_{0}(\delta)$. Then for any

(A1) $x_{1} \in \Xi(k+\log (1 / \kappa), \kappa, \delta)(c f . \S$. .1)

(A2) $I \subset \mathbb{R}$ a closed interval centered at 0 with $|I| \leq e^{\kappa_{37} k}$,

(A3) $S \subset I$ a subset with $\partial I \subset S$

there is some $s \in\left[-e^{k}, e^{k}\right]$ and a set $S^{\prime}$ with $\partial I \subset S^{\prime} \subset I$ so that:

(B1) $u(s) \cdot x_{1} \in \Xi\left(\kappa_{38} k, \tilde{\kappa}, \delta\right)$,

(B2) $\delta\left(S^{\prime} ; I\right) \leq \frac{\delta(S ; I)}{1+\kappa_{39}}$

(B3) every point of $v\left(S^{\prime}\right) u(s) \cdot x_{1}$ is within $e^{-\kappa_{40} k}$ of a point in the set $\Omega_{D} u\left(\left[-e^{k}, e^{k}\right]\right) v(S) . x_{1}$, with $\Omega_{D} \subset D$ an appropriately chosen fixed compact subset.

Here $\tilde{\kappa}, \kappa_{37}, \kappa_{38}, \kappa_{40}$ are constants depending only on $\delta$, while $\kappa_{39}$ is an absolute constant.

Proof. Set $\sigma=\log |I|$, and $\bar{I}=e^{-\sigma} I=\left[-\frac{1}{2}, \frac{1}{2}\right]$. By Proposition 4.4 applied with $T=\frac{1}{2} e^{k}$ the set

$$
F=\left\{|s| \leq \frac{1}{2} e^{k}: u_{t} \cdot x_{1} \in \Xi((1-\delta) k-10, \tilde{\kappa}, \delta)\right\}
$$


satisfies that

$$
e^{-k} m(F) \geq 1-\frac{K_{16} \tilde{\kappa}^{1 / 2}}{1-e^{-\delta / 2}}
$$

with $K_{16}$ as in Proposition 4.4 moreover, if $\tilde{\kappa}$ is sufficiently small, depending on $\delta$ the right hand side of the above equation can be made to be arbitrarily close to one. The precise choice of $\tilde{\kappa}$ will be specified later; in addition to $\delta$ it depends on the constants of Proposition 7.11 Theorem 9.3, but will not depend on any other choices we make in this proof. In particular, we assume $\tilde{\kappa}$ is sufficiently small so that $e^{-k} m(F) \geq 1 / 2$. Unless otherwise specified, all the constants $c_{i}, C_{i}$ appearing in this proof may depend on $\delta$ as well as our as yet unspecified choice of $\tilde{\kappa}{ }^{(4)}$

Let

$$
\alpha_{1}=\frac{(1-\delta)^{2}}{4+2 \delta}
$$

It follows from the lower bound above on the measure of $F$ that there is a subinterval $J \subset\left[-\frac{1}{2} e^{k}, \frac{1}{2} e^{k}\right]$ of length $e^{\alpha_{1} k}$ so that $m(J \cap F) \geq|J| / 2$. The lower bound on $m(J \cap F)$ implies that we may extract from $J \cap F$ a $R$-separated ${ }^{(5)}$ finite subset $E \subset F$ of cardinality $\geq|J| / 2 R$, with $R$ depending only on $\tilde{\kappa}$ (specifically, $R=K_{20}$ of Lemma 4.7, applied to points in $\Xi(\cdot, \tilde{\kappa}, \delta))$. Since

$$
\left\{u_{\xi} \cdot x_{1}: \xi \in E\right\} \subset \Xi((1-\delta) k-10, \tilde{\kappa}, \delta) \subset X_{\kappa}
$$

we conclude that there must be two distinct points $\xi, \xi^{\prime} \in E$ with

$$
d\left(u_{\xi} \cdot x_{1}, u_{\xi^{\prime}} \cdot x_{1}\right) \leq C_{1} e^{-\alpha_{1} k / 8}
$$

(the number 8 appears because it is the dimension of $X$ ). Write $x_{2}=$ $u_{\xi} \cdot x_{1}, x_{2}^{\prime}=u_{\xi^{\prime}} \cdot x_{1}$; assuming $C_{1} e^{-\alpha_{1} k / 8}$ is smaller than some absolute constant (equivalently, that $k$ is larger than some $k_{0}$ depending on $\delta$ ) we have that $x_{2}^{\prime}=h_{0} \cdot x_{2}$ for $h_{0}$ sufficiently close to the identity for Lemma 4.7. Moreover 10.1a) implies that

$$
\left\|h_{0}-\mathbf{e}\right\| \leq C_{2} e^{-\alpha_{1} k / 8} .
$$

On the other hand, if $R$ is sufficiently large the bound

$$
R<\left|\xi-\xi^{\prime}\right|<|J|=c_{? ?} e^{\alpha_{1} k}
$$

implies by Lemma 4.7 that we may write $h_{0}=u_{+} a_{0} u_{-}$with $u_{+} \in$ $\mathcal{U}^{+}, u_{-} \in \mathcal{U}^{-}, a_{0} \in A$ and

$$
\left\|u_{-}-\mathbf{e}\right\| \geq c_{3} e^{-\frac{2+\delta}{1-\delta} \alpha_{1} k}=c_{3} e^{-(1-\delta) k / 2} .
$$

In particular, $u_{-} \neq \mathbf{e}$ so $h_{0} \notin N_{G}(U)$.

\footnotetext{
${ }^{(4)}$ Note that in the effective proof of the Oppenheim Conjecture (including the strong, nonhomogeneous, form) $\delta$ can be chosen to be an absolute constant.

${ }^{(5)}$ I.e. $\left|\xi-\xi^{\prime}\right| \geq M$ for every distinct $\xi, \xi^{\prime} \in E$.
} 
We now wish to apply Proposition 7.11 to $x_{3}=a(-\sigma / 2) \cdot x_{2}$ and $x_{3}^{\prime}=a(-\sigma / 2) \cdot x_{2}^{\prime}$. We set the parameter $\kappa_{37}$ from the statement of the Main Lemma to be $\kappa_{37}=\frac{\alpha_{1}}{1000}$ so that

$$
\sigma \leq \alpha_{1} k / 1000 \text {. }
$$

Then $x_{3}^{\prime}=h_{1} \cdot x_{3}$ with $h_{1}=u_{+}^{\prime} a_{1} u_{-}^{\prime}$ and

$$
\begin{aligned}
\left\|u_{-}^{\prime}-\mathbf{e}\right\| & \geq\left\|u_{-}-\mathbf{e}\right\| \geq c_{3} e^{-(1-\delta) k / 2} \\
\left\|h_{1}-\mathbf{e}\right\| & \leq C_{4} e^{\sigma}\left\|h_{0}-\mathbf{e}\right\| \leq C_{5} e^{-\alpha_{1} k / 10} .
\end{aligned}
$$

By Proposition 7.11 there are

(a) $c_{6} e^{\alpha_{1} k / 40} \leq T_{2} \leq C_{7} e^{k / 2}$,

(b) a rational function $\phi$ satisfying $K_{31}^{-1} \leq \phi^{\prime}(t) \leq K_{31}$ on $\left[-T_{2}, T_{2}\right]$,

(c) polynomials $c(t), b(t)$ so that the family of affine functions $F_{t}(x)=$ $(x+b(t)) / c(t)^{2}$ satisfy

$$
\begin{aligned}
\max _{x \in \bar{I}}\left|F_{t}(x)-x\right| & \leq \kappa_{30} & & \text { for } t \in\left[-T_{2}, T_{2}\right] \\
& =\kappa_{30} & & \text { for } t=-T_{2} \text { or } T_{2}
\end{aligned}
$$

so that for every $|t| \leq T_{2}$

$$
d\left(u(t) \cdot x_{3}^{\prime}, a(\log c(t)) v\left(\frac{b(t)}{c(t)^{2}}\right) u(\phi(t)) \cdot x_{3}\right)<C_{8} e^{-\alpha_{1} k / 40} .
$$

Since for $g_{1}, g_{2} \in G$,

$$
d_{G}\left(a(\sigma / 2) g_{1}, a(\sigma / 2) g_{2}\right) \leq e^{\sigma} d_{G}\left(g_{1}, g_{2}\right),
$$

it follows that for $|t| \leq T_{1}=e^{\sigma / 2} T_{2}$ the points $x_{2}=a(\sigma / 2) \cdot x_{3}, x_{2}^{\prime}=$ $a(\sigma / 2) \cdot x_{3}^{\prime}$ satisfy

$$
d\left(u(t) \cdot x_{2}^{\prime}, a(\log \tilde{c}(t)) v\left(\frac{e^{\sigma} \tilde{b}(t)}{\tilde{c}(t)^{2}}\right) u(\tilde{\phi}(t)) \cdot x_{2}\right)<C_{8} e^{\sigma} e^{-\alpha_{1} k / 40}
$$

with $\tilde{b}(t)=b\left(e^{-\sigma / 2} t\right), \tilde{c}(t)=c\left(e^{-\sigma / 2} t\right), \tilde{\phi}(t)=\phi\left(e^{-\sigma / 2} t\right)$; set also

$$
\tilde{F}_{t}(x)=x / \tilde{c}(t)^{2}+e^{\sigma} \tilde{b}(t) / \tilde{c}(t)^{3} .
$$

Equation 10.1e implies that for $s \in I$

$$
\begin{aligned}
& d\left(v(s) u(t) \cdot x_{2}^{\prime}, a(\log \tilde{c}(t)) v\left(\tilde{F}_{t}(s)\right) u(\tilde{\phi}(t)) \cdot x_{2}\right)= \\
& \quad=d\left(v(s) u(t) \cdot x_{2}^{\prime}, a(\log \tilde{c}(t)) v\left(\frac{s+e^{\sigma} \tilde{b}(t)}{\tilde{c}(t)^{2}}\right) u(\tilde{\phi}(t)) \cdot x_{2}\right) \\
& \quad=d\left(v(s) u(t) \cdot x_{2}^{\prime}, v(s) a(\log \tilde{c}(t)) v\left(\frac{e^{\sigma} \tilde{b}(t)}{\tilde{c}(t)^{2}}\right) u(\tilde{\phi}(t)) \cdot x_{2}\right)
\end{aligned}
$$

By (10.1e) and the inequality

$$
d_{G}\left(v(s) g_{1}, v(s) g_{2}\right) \leq C_{9} s^{2} d_{G}\left(g_{1}, g_{2}\right) \quad \text { for } g_{1}, g_{2} \in G
$$


we can further estimate $(10.1 \mathrm{f})$ by

$$
\text { 10.1f }<C_{10} e^{3 \sigma} e^{-\alpha_{1} k / 40} \leq C_{10} e^{-\alpha_{1} k / 50} .
$$

Let $\alpha_{2}=\alpha_{1} / 50, T_{1}=e^{\sigma / 2} T_{2}$, and note that assuming $k_{0}$ was chosen large enough we have that

$$
T_{1}<e^{(1-\delta) k-10} .
$$

Recalling that on the interval $|t| \leq T_{1}$ we have that $\log \tilde{c}(t)$ is in some fixed finite interval, we conclude that for every $|t| \leq T_{1}$, every point of

$$
v\left(\tilde{F}_{t}(S)\right) u(\tilde{\phi}(t)) \cdot x_{2}
$$

is within distance $\leq C_{11} e^{-\alpha_{2} k}$ of some point in $\Omega_{D} u(t) v(S) \cdot x_{2}^{\prime}$, for an appropriate choice of symmetric neighborhood $\Omega_{D}$ of $\mathbf{e}$ in $D$. Since $|\phi(0)|=|\tilde{\phi}(0)|<1$ (cf. note after the statement of Proposition 7.11), by (b) above for $|t|<T_{1}$ and if $k_{0}$ is large enough,

$$
|\tilde{\phi}(t)|<K_{31}(|t|+1)<2 K_{31} e^{(1-\delta) k-10}<\frac{1}{2} e^{k},
$$

hence both $u(t) \cdot x_{2}^{\prime}$ and $u(\tilde{\phi}(t)) \cdot x_{2}$ are in $u\left(\left[-e^{k}, e^{k}\right]\right) \cdot x_{1}$. It follows that for every $|t| \leq T_{1}$ and $x \in v\left(\tilde{F}_{t}(S)\right) u(\tilde{\phi}(t)) . x_{2}$

$$
d\left(x, \Omega_{D} u\left(\left[-e^{k}, e^{k}\right]\right) v(S) \cdot x_{1}\right) \leq C_{11} e^{-\alpha_{2} k} .
$$

Since $v(S) u(\tilde{\phi}(t)) \cdot x_{2} \subset u\left(\left[-e^{k}, e^{k}\right]\right) v(S) . x_{1}$, the estimate 10.1h holds in fact for every $x \in v\left(S \cup \tilde{F}_{t}(S)\right) u(\tilde{\phi}(t)) \cdot x_{2}$.

Similarly, every point of $v\left(\tilde{F}_{t}^{-1}(S)\right) u(t) \cdot x_{2}^{\prime}$ is within distance at most $C_{11} e^{-\alpha_{2} k}$ of some point in $\Omega_{D}^{-1} u(\phi(t)) v(S) . x_{2}$ hence for every point $x^{\prime}$ in the set $v\left(S \cup \tilde{F}_{t}^{-1}(S)\right) u(t) \cdot x_{2}^{\prime}$

$$
d\left(x^{\prime}, \Omega_{D} u\left(\left[-e^{k}, e^{k}\right]\right) v(S) \cdot x_{1}\right) \leq C_{11} e^{-\alpha_{2} k} .
$$

We are now in a position to apply Theorem 9.3. Using the parameters $d, A, B, \Delta$ for that theorem that are applicable to the case at hand (in particular, $A=-2, B=2, \Delta=\kappa_{30}$ ), the constants $\kappa_{34}$ and $\kappa_{35}$ from the statement of that theorem become absolute constants and we shall treat them as such. Applying Theorem 9.3 to $F_{ \pm t / T_{2}}$ (with the sign determined according to whether equality is attained at $-T_{2}$ or $T_{2}$ in (10.1d) and $\bar{S}=e^{-\sigma} S \subset \bar{I}$, we see that there is an $\epsilon \in\{ \pm 1\}$ and subset $\bar{\Lambda}$ of $t \in\left[-T_{2}, T_{2}\right]$ of size $\geq \kappa_{35} T_{2} / 2$ so that for every $t \in \bar{\Lambda}$,

$$
\delta\left(\bar{S} \cup\left[F_{t}\right]^{\epsilon}(\bar{S}) ; \bar{I}\right) \leq \delta(\bar{S} ; \bar{I}) /\left(1+\kappa_{34}\right) .
$$

It follows that for $t \in \Lambda:=e^{\sigma} \bar{\Lambda}$ there is some set $S^{\prime}$, depending on $t$, with $\delta\left(S^{\prime} ; I\right) \leq \delta(S ; I) /\left(1+\kappa_{34}\right)$ so that for at least one of $x=u(\phi(t)) \cdot x_{2}$ or $x=u(t) \cdot x_{2}^{\prime}$

$$
\max _{s \in S^{\prime}} d\left(v(s) . x, \Omega_{D} u\left(\left[-e^{k}, e^{k}\right]\right) v(S) \cdot x_{1}\right) \leq C_{11} e^{-\alpha_{2} k} .
$$

It remains to apply Proposition 4.4 again twice. By 10.1g,

$$
(1-\delta) k-10 \geq \log T_{1},
$$


hence since $x_{2}, x_{2}^{\prime} \in \Xi(k-10, \tilde{\kappa}, \delta)$ we may apply Proposition 4.4 to conclude that the set $\Psi^{\prime}$ defined by

$$
\Psi^{\prime}=\left\{t \in\left[-T_{1}, T_{1}\right]: u_{t} \cdot x_{2}^{\prime} \notin \Xi\left((1-\delta) \log T_{1}+\log \tilde{\kappa}-10, \tilde{\kappa}, \delta\right)\right\}
$$

satisfies that

$$
m\left(\Psi^{\prime}\right) \leq \frac{2 K_{16} \tilde{\kappa}^{1 / 2}}{1-e^{-\delta / 2}} T_{1}
$$

In view of the bound $K_{31}^{-1} \leq \phi^{\prime}(t) \leq K_{31}$, Proposition 4.4 and $10.1 \mathrm{~g}$ also imply that the set

$$
\Psi^{\prime \prime}=\left\{t \in \phi\left(\left[-T_{1}, T_{1}\right]\right): u(t) \cdot x_{2} \notin \Xi\left((1-\delta) \log T_{1}-C_{12}, \tilde{\kappa}, \delta\right)\right\}
$$

for $C_{12}=\log \tilde{\kappa}-10-\log K_{31}$ satisfies the estimate

$$
m\left(\Psi^{\prime \prime}\right) \leq \frac{K_{16} \tilde{\kappa}^{1 / 2}}{\left(1-e^{-\delta / 2}\right)}\left|\phi\left(\left[-T_{1}, T_{1}\right]\right)\right| \leq \frac{2 K_{31} K_{16} \tilde{\kappa}^{1 / 2}}{\left(1-e^{-\delta / 2}\right)} T_{1},
$$

hence $\Psi=\phi^{-1}\left(\Psi^{\prime \prime}\right)$ satisfies

$$
m(\Psi) \leq \frac{2 K_{31}^{2} K_{16} \kappa^{1 / 2}}{1-e^{-\delta / 2}} T_{1}
$$

It follows that if $\tilde{\kappa}$ was chosen appropriately (i.e. $<c_{13} \delta^{2}$ for a sufficiently small absolute constant $c_{13}$ )

$$
\Lambda \backslash\left(\Psi \cup \Psi^{\prime}\right) \neq \emptyset .
$$

If $t \in \Lambda \backslash\left(\Psi \cup \Psi^{\prime}\right)$, then at least one of $x=u(\phi(t)) \cdot x_{2}$ or $x=u(t) \cdot x_{2}^{\prime}$ satisfies (10.1j) for some set $S^{\prime}$ with

$$
\delta\left(S^{\prime} ; I\right) \leq \delta(S ; I) /\left(1+\kappa_{34}\right)
$$

as well as

$$
x \in \Xi\left((1-\delta) \log T_{1}-C_{12}, \kappa, \delta\right) .
$$

Since $(1-\delta) \log T_{1}-C_{12}$ is clearly $\gg k$ (with implicit constant depending only on $\delta$ ), the Main Lemma follows.

\section{Proof of Theorem 3.8}

Recall that

$$
\delta(S ; I)=|I|^{-1} \int_{I} d_{\mathbb{R}}(x, S) d x .
$$

We note the following elementary property of $\delta(S ; I)$ :

11.1. Lemma. Let $I$ be an interval, and $S \subset I$ with $\partial I \subset S$. Then for every $t \in I$ there is a $s \in S$ with

$$
|t-s| \leq \sqrt{\delta(S ; I)}|I|
$$


Proof. Suppose not. Then there would be an interval, say $J$, of length $>2 \sqrt{\delta(S ; I)}|I|$ in $I$ with no point of $S$. But then

$$
\begin{aligned}
\delta(S ; I) & \geq|I|^{-1} \int_{J} d_{\mathbb{R}}(x, S) d x \\
& >2 \int_{0}^{\sqrt{\delta(S ; I)}} x d x \geq \delta(S ; I)
\end{aligned}
$$

in contradiction.

11.2. Proposition. Let $\delta, \kappa \in(0,1)$, and $k \geq k_{0}(\delta)$ (for an appropriate choice of $\left.k_{0}(\delta)\right)$. Then for any

(A1) $x_{1} \in \Xi(k+\log (1 / \kappa), \kappa, \delta)$

(A2) $I \subset \mathbb{R}$ a closed interval centered at 0 with $|I| \leq e^{\kappa_{37} \sqrt{k}}$,

there is some $s \in\left[-2 e^{k}, 2 e^{k}\right]$ and a set $S^{\prime}$ with $\partial I \subset S^{\prime} \subset I$ so that:

(B1) $u(s) \cdot x_{1} \in \Xi(\sqrt{k}, \tilde{\kappa}, \delta)$,

(B2) $\delta\left(S^{\prime} ; I\right) \leq k^{-\kappa_{41}}$

(B3) every point of $v\left(S^{\prime}\right) u(s) \cdot x_{1}$ is within $2 e^{-\kappa_{40} \sqrt{k}}$ of a point in the $s e(6)$

$$
B_{k^{K_{42}}}^{D} u\left(\left[-2 e^{k}, 2 e^{k}\right]\right) v(\partial I) \cdot x_{1} .
$$

Here $\tilde{\kappa}, \kappa_{37}, \kappa_{40}, \kappa_{41}, K_{42}$ depend only on $\delta$.

Proof. We apply Lemma 10.1 iteratively, starting with $x_{1}, S_{1}=\partial I$ and the given $k$. Let $T_{1}=e^{k}$. After applying the lemma once, we get a point $x_{2}=u(s) \cdot x_{1} \in \Xi\left(\kappa_{38} k, \tilde{\kappa}, \delta\right)$ with $|s|<e^{k}$ and a set $S_{2}$ with $\partial I \subset S_{2} \subset I$ so that

$$
d\left(v(\tau) \cdot x_{2}, \Omega_{D} u\left(\left[-T_{1}, T_{1}\right]\right) v(\partial I) \cdot x_{1}\right)<e^{-\kappa_{40} k} \quad \forall \tau \in S_{2} .
$$

We will now apply Lemma 10.1 again, on $x_{2}, S_{2}$ and $k_{2}=\alpha k$ for an appropriately chosen $0<\alpha<1 / 2$. In order to satisfy (A1), i.e. that $x_{2} \in \Xi\left(k_{2}, \tilde{\kappa}, \delta\right)$, we need to require that $\alpha \leq \kappa_{38}$. If we do that, we get a point $x_{3}$ and set $S_{3}$ so that

$$
d\left(v(\tau) x_{3}, \Omega_{D} u\left(\left[-e^{\alpha k}, e^{\alpha k}\right]\right) v\left(S_{2}\right) \cdot x_{2}\right)<e^{-\kappa_{40} \alpha k} \quad \forall \tau \in S_{3} .
$$

As $d(u(s) . x, u(s) . y) \ll s^{4} d(x, y)$ we can deduce from (11.2a) and 11.2b) that for any $\tau \in S_{3}$,

$$
d\left(v(\tau) x_{3}, \Omega_{D}^{2} u\left(\left[-T_{2}, T_{2}\right]\right) v(\partial I) \cdot x_{1}\right)<e^{-\kappa_{40} \alpha k}+C_{1} e^{4 \alpha k-\kappa_{40} k}
$$

with $T_{2}=e^{k}+e^{\alpha k}$. Choosing $\alpha \leq \frac{\kappa_{40}}{\left(4+2 \kappa_{40}\right)}$ and $k_{0}$ large enough, we get that the right hand side of the above equation is

$$
\leq 2 e^{-\kappa_{40} \alpha k} \text {. }
$$

${ }^{(6)}$ Recall that $B_{R}^{D}=\{h \in D:\|h-\mathbf{e}\|<R\}$. 
We continue iteratively in this way, obtaining points $x_{j} \in \Xi\left(\alpha^{j-1} k, \tilde{\kappa}, \delta\right)$ and sets $S_{j} \subset I$ with $\delta\left(S_{j} ; I\right) \leq\left(1+\kappa_{39}\right)^{-j}$ as longer as $\alpha^{j} k \geq \sqrt{k}$. Stopping at $j_{0}=\lfloor\log k / 2 \log (1 / \alpha)\rfloor$ we get the proposition, with

$$
q \delta\left(S_{j_{0}} ; I\right) \leq\left(1+\kappa_{39}\right)^{-j_{0}} \leq \alpha^{-1} k^{-\kappa_{41}}
$$

for $\kappa_{41}=\log \left(1+\kappa_{39}\right) / 2 \log \alpha$ and so that for any $\tau \in S_{j_{0}}$,

$$
d\left(v(\tau) x_{j_{0}}, \Omega_{D}^{j_{0}} u\left(\left[-T_{j_{0}}, T_{j_{0}}\right]\right) v(\partial I) . x_{1}\right)<2 e^{-\kappa_{40} \alpha^{j_{0}} k}
$$

where $T_{j_{0}}=\sum_{j=0}^{j_{0}} e^{\alpha^{j} k}<2 e^{k}$. Note that $\Omega_{D}^{j_{0}} \subset B_{C^{j_{0}}}^{D}$ for an appropriately chosen $C$ depending on $\Omega_{D}$, hence since $j_{0} \propto \log k$

$$
\Omega_{D}^{j_{0}} \subset B_{k^{K_{42}}}^{D} .
$$

Proof of Theorem 3.8. Apply Proposition 11.2 on $x_{1}$ for an interval $I$ of size $|I| \leq k^{\kappa_{41} / 4}$. Let $S^{\prime}$ be as in that proposition, in particular $\delta\left(S^{\prime} ; I\right) \leq k^{-\kappa_{41}}$. By Lemma 11.1 it follows that any $\tau \in I$ will be within $k^{-\kappa_{41} / 4}$ of a point in $S^{\prime}$, hence

$$
d\left(v(\tau) \cdot x_{1}, B_{k^{K_{42}}}^{D} u\left(\left[-2 e^{k}, 2 e^{k}\right]\right) v(\partial I) \cdot x_{1}\right) \leq C_{2}\left(2 e^{-\kappa_{40} \sqrt{k}}+k^{-\kappa_{41} / 4}\right) .
$$

\section{Applichtions to QUADRATiC FORMS}

In this section we relate Theorem 2.8 regarding effective density properties of orbits of $H$ on $G / \Gamma$ to value of quadratic forms, establishing Theorem 1.10 and Corollary 1.12 .

We note the following well-known fact, for which we include a proof (or at least a sketch of one) for completeness:

12.1. Lemma. Let $Q$ be a signature $(2,1)$ ternary quadratic form with $\operatorname{det} Q=-1$. Then there is a $g \in G$ so that $Q=Q_{0} \circ g$ with

$$
[g]_{\Gamma} \in X_{\rho} \quad \rho=c_{1}\|Q\|^{K_{43}} .
$$

Here, as we have done throughout this paper, $Q_{0}$ denotes the quadratic form $y^{2}-2 x z$.

Proof. Following a similar procedure as in $\$ 7.2$, it is easy to find a basis $v_{1}, v_{2}, v_{3}$ of $\mathbb{R}^{3}$ so that

$$
\begin{gathered}
Q\left(v_{1}\right)=Q\left(v_{3}\right)=Q\left(v_{1}, v_{2}\right)=Q\left(v_{2}, v_{3}\right)=0 \\
Q\left(v_{2}\right)=1 \quad Q\left(v_{1}, v_{3}\right)=-2
\end{gathered}
$$

with $\left\|v_{i}\right\|$ all controlled polynomially by $\|Q\|$. Indeed, we choose $v_{1}$ to be any unit vector isotropic for $Q$ (i.e. such that $Q\left(v_{1}\right)=0$ ), take

$$
v_{3}=-2\left\|Q v_{1}\right\|^{-2} Q v_{1}+\lambda v_{1}
$$


with $\lambda$ chosen so that $Q\left(v_{3}, v_{3}\right)=0$ (here we identified $Q$ with the matrix representing it, so $Q v_{1}$ is a vector in $\mathbb{R}^{3}$ ) and $v_{2}$ a vector satisfying the three linear equations

$$
Q\left(v_{1}, v_{2}\right)=Q\left(v_{3}, v_{2}\right)=0, \quad \operatorname{det}\left(v_{1}, v_{2}, v_{3}\right)=0 .
$$

The norm of $\left\|v_{3}\right\|$ can be easy controlled in terms of $\|Q\|$ and $\left\|Q^{-1}\right\| \ll$ $\|Q\|^{2}$. In order to be able to control $\left\|v_{2}\right\|$ we need to show that the one-dimensional subspace of $\mathbb{R}^{3}$ defined by $Q\left(v_{1}, x\right)=Q\left(v_{3}, x\right)=0$ is bounded away from the subspace generated by $v_{1}$ and $v_{2}$. If $x$ is in this one-dimensional subspace

$$
Q\left(v_{i}, x-a_{1} v_{1}-a_{2} v_{2}\right)=-2 a_{4-i} \quad \text { for } i=1,3
$$

hence

$$
\begin{gathered}
\left\|x-a_{1} v_{1}-a_{2} v_{2}\right\| \geq \max \left(2\|Q\|^{-1}\left|a_{1}\right|, 2\|Q\|^{-1}\left|a_{3}\right|,\right. \\
\left.\|x\|-\left|a_{1}\right|\left\|v_{1}\right\|-\left|a_{3}\right|\left\|v_{3}\right\|\right)
\end{gathered}
$$

hence

$$
\left\|x-a_{1} v_{1}-a_{2} v_{2}\right\| \gg\|x\| \min \left(\left\|v_{1}\right\|^{-1},\left\|v_{3}\right\|^{-1}\right) .
$$

Setting $h$ to be the matrix formed by the column vectors $v_{1}, v_{2}, v_{3}$, equation (12.1a) implies that $Q_{0}=Q \circ h$, i.e. $Q=Q_{0} \circ\left(h^{-1}\right)$. If $w \in h^{-1} \mathbb{Z}^{3}$, for at least one $i$ we have that $\left(w, v_{i}\right) \in \mathbb{Z} \backslash 0$ hence $\|w\| \geq\left\|v_{i}\right\|^{-1}$.

12.2. Proof of Theorem 1.10. Let $Q_{1}$ be an indefinite, ternary quadratic form as in the statement of the theorem. By Lemma 12.1, there is a $g_{1} \in G$ so that $Q_{1}=Q_{0} \circ g_{1}$ and $\left[g_{1}\right]_{\Gamma} \in X_{\rho}$ with $\rho \gg\left\|Q_{1}\right\|^{K_{43}}$. It also follows from the proof of that lemma that $\left\|g_{1}^{-1}\right\| \ll\left\|Q_{1}\right\|^{K_{43}}$.

Applying Theorem 2.8 on $x_{1}=\left[g_{1}\right]_{\Gamma}$ for the given $T$ we may conclude that either there is a point $x_{2} \in G / \Gamma$ with $H . x_{2}$ periodic and with $\operatorname{disc}_{Q}\left(H . x_{2}\right)<T^{3 \epsilon}$ and $d\left(x_{1}, x_{2}\right) \leq T^{-1}$ or (ii) of Theorem 2.8 holds.

In the former case, writing $x_{2}=\left[g_{2}\right]_{\Gamma}$ with $d_{G}\left(g_{1}, g_{2}\right) \leq T^{-1}$, we may conclude that the integral form

$$
Q_{2}=\lambda\left(Q_{0} \circ g_{2}\right) \quad \lambda=\operatorname{disc}_{Q}\left(H \cdot x_{2}\right)^{1 / 3}
$$

satisfies that

$$
\left\|Q_{1}-\lambda^{-1} Q_{2}\right\| \ll\left\|Q_{1}\right\| d\left(g_{1}, g_{2}\right),
$$

establishing (i) of Theorem 1.10 .

If (ii) of Theorem 2.8 holds we have a $x_{2}=\left[g_{2}\right]_{\Gamma}=h_{1} . x_{1} \in X_{\kappa_{6}}$ with $h_{1} \in B_{T^{K_{5}}}^{H_{5}}$ so that for every $s \in\left[-\log T^{\kappa_{7}}, \log T^{\kappa_{7}}\right]$ the point $v(s) \cdot x_{2}$ is within $\log T^{-\kappa_{7}}$ of a point in the set $B_{T^{K_{5}}}^{H} . x_{1}$.

As $x_{2} \in X_{\kappa_{6}}$, for appropriate $c_{1}, C_{2}>0$, there will be a vector $\mathbf{w}=\left(w_{1}, w_{2}, w_{3}\right)^{\top} \in g_{2} \mathbb{Z}^{3}$ with

$$
c_{1} \leq w_{3} \leq\|\mathbf{w}\| \leq C_{2} .
$$


Write $\mathbf{w}=g_{2} \mathbf{n}$ with $\mathbf{n} \in \mathbb{Z} \backslash\{0\}$ and let $q_{0}=Q_{0}(\mathbf{w})$. Then for any $s \in \mathbb{R}$

$$
Q_{0}(v(s) \cdot \mathbf{w})=q_{0}-2 s w_{3}^{2} .
$$

If $s \in\left[-\log T^{\kappa_{7}}, \log T^{\kappa_{7}}\right]$ there will be some $h_{(s)} \in H$ with $\left\|h_{(s)}\right\|<$ $T^{K_{5}}$ so that $d\left(v(s) \cdot x_{2}, h_{(s)} \cdot x_{1}\right)<\log T^{\kappa_{7}}$. It follows that in the lattice corresponding to $h_{(s)} \cdot x_{1}$ there should be a vector $\mathbf{w}_{(s)}=h_{(s)} g_{1} \mathbf{m}_{(s)}$ satisfying

$$
\begin{aligned}
\left\|\mathbf{w}_{(s)}-v(s) . \mathbf{w}\right\| & \ll(\log T)^{-\kappa_{7}}\|v(s) \cdot \mathbf{w}\| \\
& \ll(\log T)^{-\kappa_{7}}(1+|s|) .
\end{aligned}
$$

From this it follows that

$$
\left|Q_{0}\left(\mathbf{w}_{(s)}\right)-q_{0}+2 s w_{3}^{2}\right| \ll(\log T)^{-\kappa_{7}}\left(1+|s|^{2}\right) .
$$

Note also that

$$
\begin{aligned}
\left\|\mathbf{m}_{(s)}\right\| & \leq\left\|g_{1}^{-1}\right\|\left\|h_{(s)}^{-1}\right\|\left\|\mathbf{w}_{(s)}\right\| \\
& \leq C_{3}\left\|Q_{1}\right\|^{K_{43}} T^{K_{5}} .
\end{aligned}
$$

As $T \geq T_{0}(\epsilon)\left\|Q_{1}\right\|^{K_{1}}$, then assuming $K_{1}$ is large enough, 12.2a $\leq$ $T^{1.1 K_{5}}$. It follows that for any $s \in\left[-\log T^{\kappa_{7} / 3}, \log T^{\kappa_{7} / 3}\right]$ there is a $\mathbf{m}_{(s)} \in \mathbb{Z}^{3}$ with $\left\|\mathbf{m}_{(s)}\right\| \leq T^{1.1 K_{5}}$ and

$$
Q_{1}\left(\mathbf{m}_{(s)}\right)=Q_{0} \circ g_{1}\left(\mathbf{m}_{(s)}\right)=Q_{0} \circ\left(h_{(s)} g_{1}\right)\left(\mathbf{m}_{(s)}\right)=Q_{0}\left(\mathbf{w}_{(s)}\right)
$$

so that

$$
\min \left\{\left|Q_{1}(\mathbf{m})-q_{0}-2 s w_{3}^{2}\right|: \mathbf{m} \in \mathbb{Z}^{3},\|\mathbf{m}\|<T^{1.1 K_{5}}\right\} \leq C_{4} \log T^{-\kappa_{7} / 3} .
$$

As $\left|q_{0}\right| \leq 2 C_{2}^{2}$ and $\left|w_{3}\right| \geq c_{1}$, assuming that $T_{0}$ is large enough so that $c_{1}^{2} \log T^{\kappa_{7} / 3}>2 C_{2}$, it follows that for $c_{5}=\frac{1}{2} c_{1}^{2}$

$\max _{|\xi| \leq c_{5} \log T^{\frac{\kappa_{7}}{3}}} \min \left\{\left|Q_{1}(\mathbf{m})-\xi\right|: \mathbf{m} \in \mathbb{Z}^{3},\|\mathbf{m}\|<T^{1.1 K_{5}}\right\} \leq C_{4} \log T^{-\kappa_{7} / 3}$, proving the theorem.

12.3. Before we prove Corollary 1.12 , we recall some basic properties of the logarithmic height of algebraic numbers.

Let $K$ be a number field, i.e. a finite extension of $\mathbb{Q}$ with $d=[K: \mathbb{Q}]$. If $\alpha \in K$, its logarithmic height height $(\alpha)$ is defined to be

$$
\operatorname{height}(\alpha)=\frac{1}{d} \sum_{v} \log \left(\max \left(1,|\alpha|_{v}^{d_{v}}\right)\right)
$$

where the summation is over all valuations of $K$, with the normalization that if $K_{v}$ is the completion of $K$ with respect to $v$ then $|\cdot|_{v}$ restricted to $\mathbb{Q}$ reduces to the ordinary $p$-adic or Euclidean absolute value; in these cases we say $v$ corresponds to the place $w=p$ or $\infty$ of $\mathbb{Q}$ respectively, and set $d_{v}=\left[K_{v}: \mathbb{Q}_{w}\right]$. In particular, if $K_{v} \cong \mathbb{R}$ then $d_{v}=1$ while if $K_{v} \cong \mathbb{C}$ then $d_{v}=2$. For more details, see [BG2, §1.3] (though 
the reader is warned that a different normalization is used there). The following basic properties of height will be useful:

$$
\begin{aligned}
\operatorname{height}\left(\frac{p}{q}\right) & =\log (\max (|p|, q)) \quad \text { for } \frac{p}{q} \in \mathbb{Q} \\
\operatorname{height}(\alpha) & =\operatorname{height}\left(\alpha^{-1}\right) \\
\operatorname{height}(\alpha \beta) & \leq \operatorname{height}(\alpha)+\operatorname{height}(\beta) \\
|\alpha|_{v} & \leq e^{d \operatorname{height}(\alpha)} \quad \text { for any valuation } v
\end{aligned}
$$

We also recall that in this normalization the height is independent of the field in which it is evaluated.

We shall make use of the following bound of Liouville type:

12.4. Lemma (cf. BG2, Thm. 1.5.21]). Let $K$ be a number field of degree $d$ and $\alpha_{1}, \alpha_{2}$ distinct elements of $K$. Let $v$ be any valuation of $K$ corresponding to $w=p$ or $\infty$ on $\mathbb{Q}$. Then

$$
\left|\alpha_{1}-\alpha_{2}\right|_{v} \geq\left(2 e^{\operatorname{height}\left(\alpha_{1}\right)+\operatorname{height}\left(\alpha_{2}\right)}\right)^{-d / d_{v}}
$$

with $d_{v}=\left[K_{v}: \mathbb{Q}_{w}\right]$.

12.5. Proof of Corollary 1.12. To deduce Corollary 1.12 from Theorem 1.10 when needs to show two things: that $\left\|Q_{1}\right\|$ can be bounded in terms of the heights and degrees of its coefficients (which is immediate from (12.3a), and that for an appropriate choice of $\epsilon>0$ case (i) of Theorem 1.10 leads to contradiction.

Suppose the coefficients of $Q_{1}$ are algebraic, but that $Q_{1}$ is not proportional to an integral form. Then there are two nonzero coefficients, say $\alpha_{1}, \alpha_{2}$, of $Q_{1}$ with $\alpha_{1} / \alpha_{2} \notin \mathbb{Q}$. Let $h_{1}, h_{2}$ be the logarithmic heights of $\alpha_{1}, \alpha_{2}$ respectively, and $d=\left[\mathbb{Q}\left(\alpha_{1}, \alpha_{2}\right): \mathbb{Q}\right]$. Assume that there is an integral form $Q_{2}$ as in (i) of Theorem 1.10 .

It follows that there is a $\lambda \geq T^{-\epsilon / 3}$ and integers $n_{1}, n_{2}$ so that $\left|\alpha_{i}-\lambda n_{i}\right|<\left\|Q_{1}\right\| T^{-1}$. Moreover, if $T>10\left\|Q_{1}\right\| \min \left|\alpha_{i}\right|^{-1}$ both $n_{1}, n_{2}$ are nonzero, hence

$$
\left|\frac{\alpha_{1}}{n_{1}}-\frac{\alpha_{2}}{n_{2}}\right| \leq 2\left\|Q_{1}\right\| T^{-1}
$$

Clearly also

$$
\left|n_{i}\right|<2 \lambda^{-1}\left\|Q_{1}\right\|
$$

However, by Lemma 12.4 .

$$
\begin{aligned}
\left|\frac{\alpha_{1}}{n_{1}}-\frac{\alpha_{2}}{n_{2}}\right| & \geq\left(2 e^{\operatorname{height}\left(\alpha_{1} / n_{1}\right)+\operatorname{height}\left(\alpha_{2} / n_{2}\right)}\right)^{-d} \\
& =\left(2 e^{\operatorname{height}\left(\alpha_{1}\right)+\operatorname{height}\left(\alpha_{2}\right)}\right)^{-d}\left(n_{1} n_{2}\right)^{-d} \\
& \geq\left(8 e^{\operatorname{height}\left(\alpha_{1}\right)+\operatorname{height}\left(\alpha_{2}\right)}\right)^{-d}\left\|Q_{1}\right\|^{-2 d} \lambda^{-2 d} .
\end{aligned}
$$

As $\lambda \geq T^{-\epsilon / 3}$, this leads to contradiction if $\epsilon<\frac{3}{2 d}$ and $T$ is large enough. 


\section{REFERENCES}

[BG1] V. Bentkus and F. Götze, Lattice point problems and distribution of values of quadratic forms, Ann. of Math. (2) 150 (1999), no. 3, 977-1027. MR:1740988 (2001b:11087)

[BG2] E. Bombieri and W. Gubler, Heights in Diophantine geometry, New Mathematical Monographs, vol. 4, Cambridge University Press, Cambridge, 2006. MR2216774 (2007a:11092)

[C] J. W. S. Cassels, Rational quadratic forms, London Mathematical Society Monographs, vol. 13, Academic Press Inc., London, 1978. MR522835 (80m:10019)

[CSD] J. W. S. Cassels and H. P. F. Swinnerton-Dyer, On the product of three homogeneous linear forms and the indefinite ternary quadratic forms, Philos. Trans. Roy. Soc. London. Ser. A. 248 (1955), 73-96. MR17,14f

[D1] S. G. Dani, Invariant measures of horospherical flows on noncompact homogeneous spaces, Invent. Math. 47 (1978), no. 2, 101-138. MR0578655 $(58$ \#28260)

[D2] S. G. Dani, On uniformly distributed orbits of certain horocycle flows, Ergodic Theory Dynamical Systems 2 (1982), no. 2, 139-158 (1983). MR693971 (84g:58068)

[D3] S. G. Dani, On orbits of unipotent flows on homogeneous spaces. II, Ergodic Theory Dynam. Systems 6 (1986), no. 2, 167-182. MR857195 (88e:58052)

[D4] S. G. Dani, A proof of Margulis' theorem on values of quadratic forms, independent of the axiom of choice, Enseign. Math. (2) 40 (1994), no. 1-2, 49-58. MR1279060 (95e:11075)

$[\mathrm{DH}]$ H. Davenport and H. Heilbronn, On indefinite quadratic forms in five variables, J. London Math. Soc. 21 (1946), 185-193. MR0020578 (8,565e)

[DM1] S. G. Dani and G. A. Margulis, Values of quadratic forms at primitive integral points, Invent. Math. 98 (1989), no. 2, 405-424. MR1016271 $(90 \mathrm{k}: 22013 \mathrm{~b})$

[DM2] S. G. Dani and G. A. Margulis, Orbit closures of generic unipotent flows on homogeneous spaces of SL(3, R), Math. Ann. 286 (1990), no. 1-3, 101128. MR1032925 (91k:22026)

[DM3] S. G. Dani and G. A. Margulis, Values of quadratic forms at integral points: an elementary approach, Enseign. Math. (2) 36 (1990), no. 1-2, 143-174. MR $91 \mathrm{k}: 11053$

[DM4] S. G. Dani and G. A. Margulis, Limit distributions of orbits of unipotent flows and values of quadratic forms, I. M. Gel'fand seminar, 1993, pp. 91137. MR1237827 (95b:22024)

[ELMV] M. Einsiedler, E. Lindenstrauss, P. Michel, and A. Venkatesh, The distribution of periodic torus orbits on homogeneous spaces, Duke Math. J. 148 (2009), no. 1, 119-174.

[EMV] M. Einsiedler, G. Margulis, and A. Venkatesh, Effective equidistribution for closed orbits of semisimple groups on homogeneous spaces, Inventiones mathematicae 177 (2009), no. 1, 137-212.

[GM] F. Götze and G. A. Margulis, Distribution of Values of Quadratic Forms at Integral Points, ArXiv e-prints (Apr. 2010), available at 1004.5123.

[KM] D. Y. Kleinbock and G. A. Margulis, Flows on homogeneous spaces and Diophantine approximation on manifolds, Ann. of Math. (2) 148 (1998), no. 1, 339-360. MR99j:11083

[M1] G. A. Margulis, The action of unipotent groups in a lattice space, Mat. Sb. (N.S.) 86(128) (1971), 552-556. MR0291352 (45 \#445) 
[M2] G. A. Margulis, Discrete subgroups and ergodic theory, Number theory, trace formulas and discrete groups (oslo, 1987), 1989, pp. 377-398. MR.90k:22013a

[M3] G. A. Margulis, Formes quadratriques indéfinies et flots unipotents sur les espaces homogènes, C. R. Acad. Sci. Paris Sér. I Math. 304 (1987), no. 10, 249-253. MR 882782 (88f:11027)

[M4] G. A. Margulis, Dynamical and ergodic properties of subgroup actions on homogeneous spaces with applications to number theory, Proceedings of the International Congress of Mathematicians, Vol. I, II (Kyoto, 1990), 1991, pp. 193-215. MR1159213 (93g:22011)

[M5] A. Mohammadi, A special case of effective equidistribution with explicit constants, Ergodic Theory Dynam. Systems 32 (2012), no. 1, 237-247. MR2873169

[MT1] G. A. Margulis and G. M. Tomanov, Invariant measures for actions of unipotent groups over local fields on homogeneous spaces, Invent. Math. 116 (1994), no. 1-3, 347-392. MR95k:22013

[MT2] G. A. Margulis and G. M. Tomanov, Measure rigidity for almost linear groups and its applications, J. Anal. Math. 69 (1996), 25-54. MR1428093 (98i:22016)

[R1] M. Ratner, Horocycle flows, joinings and rigidity of products, Ann. of Math. (2) 118 (1983), no. 2, 277-313. MR85k:58063

[R2] M. Ratner, On measure rigidity of unipotent subgroups of semisimple groups, Acta Math. 165 (1990), no. 3-4, 229-309. MR91m:57031

[R3] M. Ratner, Strict measure rigidity for unipotent subgroups of solvable groups, Invent. Math. 101 (1990), no. 2, 449-482. MR92h:22015

[R4] M. Ratner, On Raghunathan's measure conjecture, Ann. of Math. (2) 134 (1991), no. 3, 545-607. MR93a:22009

[R5] M. Ratner, Raghunathan's topological conjecture and distributions of unipotent flows, Duke Math. J. 63 (1991), no. 1, 235-280. MR93f:22012

[S] N. A. Shah, Unipotent flows on products of $\mathrm{SL}(2, K) / \Gamma$ 's, Dynamical systems and Diophantine approximation, 2009, pp. 69-104. MR2808404 (2012m:22020)

Einstein Institute of Mathematics, The Hebrew University of Jerusalem, Jerusalem, 91904, IsRAeL

Yale University Mathematics Dept. PO Box 208283 New Haven, CT 06520, USA 